\title{
BSA-assisted synthesis of ultrasmall gallic acid-Fe(III) coordination polymer nanoparticles for cancer theranostics
}

This article was published in the following Dove Press journal:

International Journal of Nanomedicine

3 October 2017

Number of times this article has been viewed

\section{Xueling $\mathrm{Mu}^{\mathrm{I}-3}$ \\ Chenglin Yan ${ }^{1-3}$ \\ Qiwei Tian ${ }^{1-3}$ \\ Jiaomin $\operatorname{Lin}^{1-3}$ \\ Shiping Yang ${ }^{1-3}$}

'Key Laboratory of Resource

Chemistry, ${ }^{2}$ Key Laboratory of Rare

Earth Functional Materials, ${ }^{3} \mathrm{Key}$

Laboratory of Molecular Imaging

Probes and Sensors, Shanghai Normal

University, Shanghai, China
Correspondence: Qiwei Tian; Shiping Yang

Shanghai Normal University, 100 Guilin

Road, Shanghai 200234, China

Tel/fax +86 2l 64322343

Email qiweitian@shnu.edu.cn;

shipingy@shnu.edu.cn

\begin{abstract}
Protein-related nanotheranostic agents hold great promise as tools to serve many clinical applications. Proteins such as BSA are used to regulate the synthesis of nondegradable inorganic nanoparticles (NPs). To fully employ the potential of such proteins, a new type of biosafe nanotheranostic agent must be designed to optimize BSA as a biomineralization agent. Here, a straightforward BSA-assisted biomineralization method was developed to prepare gallic acid (GA)-Fe(III) coordination polymer NPs. BSA-coated GA-Fe (GA-Fe@BSA) NPs were ultrasmall $(3.5 \mathrm{~nm})$ and showed good biocompatibility, a lower $r_{2}: r_{1}$ ratio (1.06), and strong absorption in the visible near-infrared region. $T_{1}$-weighted magnetic resonance imaging of tumor-bearing mice before and after intratumoral injection with GA-Fe@BSA NPs definitively demonstrated positive change. In a subsequent in vivo study, antitumor activity was precipitated by intratumoral injection of GA-Fe@BSA NPs combined with laser treatment, suggesting excellent outcomes with this treatment method. These results describe a successful protocol in which BSA regulated the synthesis of benign organic polymer NPs. GA-Fe@BSA NPs have the potential to be ideal agents to be used in clinical theranostic nanoplatforms.
\end{abstract}

Keywords: BSA, MRI, gallic acid, coordination polymer, theranostics

\section{Introduction}

Nanotheranostic agents have attracted much interest, given the potential of nanoengineering for cancer detection and treatment. ${ }^{1}$ This is especially true of those agents that integrate both imaging and therapeutic functions into one nanoparticle (NP) ${ }^{2,3}$ The development of nanotheranostic agents can be classified into two categories: inorganic and organic. ${ }^{4}$ Inorganic nanotheranostic agents, including noble metals ${ }^{5,6}$ semiconductor NPs,,${ }^{7,8}$ and carbon materials, ${ }^{9-11}$ are studied extensively, as they are highly stable and have multifunctional properties. However, inorganic materials do not degrade, and long-term retention in the body limits their clinical use for cancer treatments, ${ }^{12}$ since agents must completely clear the body within a reasonable period to receive regulatory approval from the US Food and Drug Administration (FDA). ${ }^{13}$ Given the pitfalls of inorganic materials, an increasing number of organic nanotheranostic platforms are under intense investigation. Organic platforms contain benign ingredients and are easily degraded, ${ }^{14-16}$ thus offering higher biological compatibility. ${ }^{17,18}$ Indeed, several protein-related theranostic agents have been approved by the FDA for clinical use, including the albumin-paclitaxel NP Abraxane ( $n a b$-paclitaxel; Celgene, Summit, NJ, USA). ${ }^{19,20}$ Therefore, the development of degradable organic and protein-related nanotheranostic agents holds great promise for clinical applications. 
Proteins, such as HSA and BSA, are enriched in N-terminal amines and cysteine residues. ${ }^{21}$ These sites exhibit strong coordination abilities with transition-metal ions, such as $\mathrm{Au}^{3+}, \mathrm{Cu}^{2+}, \mathrm{Gd}^{3+}$, and $\mathrm{Bi}^{3+} .{ }^{22}$ Research has shown a significant difference between the functions of HSA and BSA. ${ }^{23}$ The high homology and low cost of BSA makes it an appropriate research proxy for HSA. Therefore, BSA is widely used as a surfactant to control the synthesis of transition-metal ion-based NPs, a process similar to the biomineralization observed in nature. ${ }^{24}$ BSA was first used by Burt et al to prepare gold NPs using direct conjugation methods. ${ }^{25}$ Several kinds of noble metals, ${ }^{26}$ metal alloys, ${ }^{27}$ and metal sulfide NPs ${ }^{28}$ have also been created using BSA as the surfactant. Recently, BSA was used as a nanoreactor to prepare theranostic agents. ${ }^{22}$ This improved biological compatibility and controlled size. These agents achieved more efficient cancer-detection rates and better treatments in vivo. For example, cypate-grafted $\mathrm{BSA}-\mathrm{Gd}_{2} \mathrm{O}_{3} \mathrm{NPs}$ were applied to near-infrared (NIR) fluorescence/photoacoustic/magnetic resonance imaging (MRI) and photothermal therapy. ${ }^{29}$ Also, BSA-stabilized $\mathrm{Bi}_{2} \mathrm{~S}_{3}$ NPs have been used for single-photonemission computed tomography/computed tomography/ photoacoustic imaging-guided combined photothermal and radiotherapies. ${ }^{28}$ Nevertheless, BSA is typically designed to regulate the synthesis of nondegradable inorganic NPs. ${ }^{25-29}$ Further research is required to determine the potential of BSA in preparation of a new kind of biological safety material, such as a coordination polymer.

Coordination polymer NPs, a nanoscale metal-organic framework, are considered an ideal platform for developing nanomedicine. These NPs have the combined advantages of both nanomaterial and organic complexes, eg, biodegradability, compositional diversity, and ease of preparation. ${ }^{30}$ As such, it was important to investigate whether BSA were able to tune the synthesis of coordination polymer NPs by the formation of a BSA-transition metal ion complex. In this process, the transition-metal ions would play a similar role as inorganic NPs. ${ }^{23}$ In a recent proof-of-concept experiment, gallic acid (GA)-Fe(III) coordination polymer NPs were explored as theranostic agents, given their strong absorbance in the NIR region and good MRI properties. ${ }^{31,32}$ Here, GA-Fe was used to investigate the role of BSA in tuning the synthesis of coordination polymer NPs.

In this report, a straightforward BSA-assisted biomineralization method is described for the preparation of GA-Fe coordination polymer NPs (Scheme 1). The formation
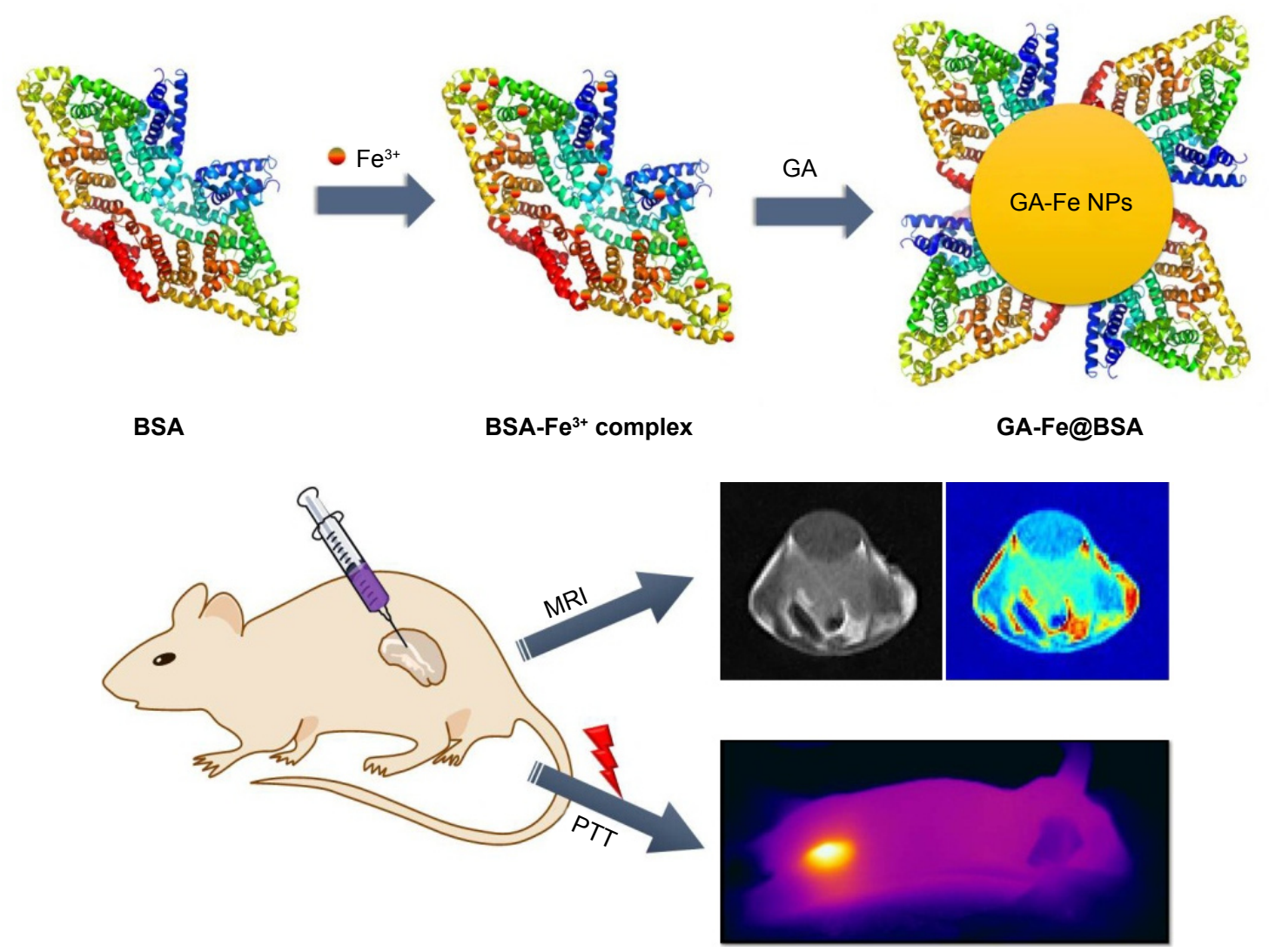

Scheme I Synthesis protocol for GA-Fe@BSA NPs and application of GA-Fe@BSA NPs in in vivo MRI and PTT for cancer. Abbreviations: GA, gallic acid; NPs, nanoparticles; MRI, magnetic resonance imaging; PTT, photothermal therapy. 
process of GA-Fe coordination polymer NPs consisted of two procedures. First, the BSA-Fe ${ }^{3+}$ complex was prepared. BSA is enriched in N-terminal amines and cysteine residues. ${ }^{21}$ These sites strongly coordinate with transition-metal ions. ${ }^{21}$ The BSA-Fe ${ }^{3+}$ complex was formed easily by thoroughly mixing an aqueous solution of $\mathrm{BSA}$ and $\mathrm{FeCl}_{3}$. Second, BSA-coated GA-Fe (GA-Fe@BSA) NPs were synthesized. GA monomers were mixed with the BSA-Fe ${ }^{3+}$ complex. GA-Fe@BSA NPs were formed by phenolic groups and carboxylic groups in GA chelating with $\mathrm{Fe}^{3+}$. GA-Fe@ BSA NPs were purified using ultrafiltration centrifugation to remove excess BSA. GA-Fe@BSA NPs were ultrasmall $(3.5 \mathrm{~nm})$ and showed good biocompatibility, lower $r_{2}: r_{1}$ ratio (1.06). and strong absorption in the visible NIR region. These properties made the GA-Fe NPs an excellent tool to enhance MRI diagnosis and photothermal therapy (Scheme 1). Results presented here describe a model that used BSA to regulate the synthesis of benign organic polymer NPs. They further support the potential of novel GA-Fe@BSA NPs as ideal agents for theranostic nanoplatform applications.

\section{Materials and methods Materials}

GA (99\%) and Fe(III) chloride hexahydrate were ordered from Sinopharm Chemical Reagent (Shanghai, China). BSA was ordered from Amresco (Solon, OH, USA). All reagents were used without further purification. Mouse selection, methods of care, welfare, and killing were approved by the animal ethics committee of Shanghai Normal University and in strict accordance with the policy of the Institutional Animal Care and Use Committee.

\section{Synthesis of GA-Fe@BSA NPs}

Using standard procedures, $66.6 \mathrm{mg}$ BSA was dissolved into $9 \mathrm{~mL}$ deionized water with stirring to form a homogeneous solution. Aqueous $\mathrm{FeCl}_{3}$ solution $(108.2 \mu \mathrm{L}, 0.1 \mathrm{~g} / \mathrm{mL})$ was added dropwise. After being stirred for 30 minutes at room temperature, a yellow, homogeneous solution was formed. Next, GA aqueous solution ( $1.02 \mathrm{~mL}, 10 \mathrm{mg} / \mathrm{mL}$ ) was added dropwise with vigorous stirring. After 3 hours' incubation, GA-Fe@BSA NPs were collected using ultrafiltration centrifugation (polyether sulfone film) with a molecular weight cut point of $100 \mathrm{kDa}$ at 3,500 rpm for 5 minutes, and then washed twice with water. The GA-Fe@BSA NPs were dissolved and stored in water at $4^{\circ} \mathrm{C}$.

\section{Characterization of GA-Fe@BSA NPs}

Sizes and morphologies of the GA-Fe@BSA NPs were measured using atomic force microscopy (NanoScope IIa; Digital
Instruments, Tonawanda, NY, USA). Fourier-transform IR (FT-IR) spectra were measured using potassium bromide pressed pellets on a Nicolet Avatar 370 FT-IR spectrophotometer. Visible NIR absorption spectra were gathered on a Beckman Coulter DU 730 ultraviolet (UV)-visible spectrophotometer using quartz cuvettes with an optical path of $1 \mathrm{~cm}$. Hydration size was recorded using a Malvern Zetasizer Nano ZS. Iron-ion concentrations were calculated with high-dispersion inductively coupled plasma atomic emission spectroscopy (Prodigy; Teledyne Leeman Labs). Magnetic resonance relaxometry was carried out on an NMI20 Analyst (Niumag, Shanghai, China).

\section{Measurement of photothermal performance}

To determine the photothermal performance of GA-Fe@ BSA NPs, temperature changes were measured with different concentrations of GA-Fe@BSA NP aqueous mixtures (pure water, 0.03, 0.06, 0.12, 0.25, 0.5, and $1 \mathrm{mM}$ ) using an FLIR A300 thermal camera and $808 \mathrm{~nm}$ laser $\left(1 \mathrm{~W} / \mathrm{cm}^{2}\right)$ ablation for 15 minutes. In photothermal stability tests, GA-Fe@BSA NP aqueous mixtures $(1 \mathrm{mM})$ were treated with eight cycles of 15-minutes laser exposure followed by 15-minutes rest.

\section{In vitro biocompatibility}

The cytotoxicity of GA-Fe@BSA NPs was evaluated on human macrophages (Shanghai Institutes for Biological Sciences) using a CCK-8 assay (Beyotime, Haimen, China). For 12- and 24-hour exposure groups, $100 \mu \mathrm{L}$ suspensions of human macrophages $\left(10^{5} / \mathrm{mL}\right)$ were seeded onto 96-well plates. Aliquots $(100 \mu \mathrm{L})$ of PBS or media containing different concentrations of GA-Fe@BSA NPs $(10,25,50$, $100,200 \mu \mathrm{M}$ ) were added. Cells were incubated for 12 or 24 hours. Next, $10 \mu \mathrm{L}$ CCK-8 was introduced to each well. After 5 minutes' incubation at $37^{\circ} \mathrm{C}$, absorbance at $450 \mathrm{~nm}$ was recorded with a microplate reader (Varioskan Flash; Thermo Fisher Scientific).

Hemolysis assays were carried out on red blood cells (RBCs; obtained from healthy BALB/c nude mice 5-6 weeks old; Shanghai Laboratory Animal Center) to evaluate the biocompatibility of GA-Fe@BSA NPs. Briefly, $0.4 \mathrm{~mL}$ of packed $\mathrm{RBCs}$ ( $2 \%$ volume ratio) was added to $1 \mathrm{~mL}$ deionized water, PBS, or PBS mixtures containing concentrations of GA-Fe@BSA NPs $(50,100,150$, and $200 \mu \mathrm{M})$ in $1.5 \mathrm{~mL}$ polyethylene tubes. PBS and deionized water were used as positive and negative controls, respectively. After 1 hour's incubation at $37^{\circ} \mathrm{C}$, mixtures were centrifuged at $3,000 \mathrm{rpm}$ for 5 minutes. Supernatant-absorbance measurements at 
$576 \mathrm{~nm}$ were taken with a Beckman Coulter DU 730 UVvisible spectrophotometer. Percentages of hemolysis were calculated using the equation $\left(\mathrm{A}_{\mathrm{t}}-\mathrm{A}_{\mathrm{nc}}\right) /\left(\mathrm{A}_{\mathrm{pc}}-\mathrm{A}_{\mathrm{nc}}\right) \times 100 \%$, where $A_{t}, A_{p c}$ and $A_{n c}$ are the absorbance of the test, positive controls, and negative controls, respectively, at $576 \mathrm{~nm}$.

Cell-cycle assays were measured using a cell-cycle assay kit (Beyotime) with strict adherence to the manufacturer's instructions. In brief, HEK293T cells (Shanghai Institutes for Biological Sciences) were plated onto a six-well plate at a density of $2 \times 10^{5}$ cells/well overnight. Next, cells were treated with GA-Fe@BSA NPs for 24 hours at a $200 \mu \mathrm{M}$ concentration. Cells were then harvested, washed twice with PBS, and analyzed with flow cytometry.

Routine blood tests were carried out to assess toxicity in GA-Fe@BSA NP-treated mice. Three healthy BALB/c mice (Shanghai Laboratory Animal Center) each received a single intravenous injection of saline (control) or GA-Fe@, BSA NPs $(200 \mu \mathrm{L}, 20 \mathrm{mM})$. After a 3-day incubation period, animals were humanely killed and dissected. Blood samples were collected from the mice and then separated into blood cells and plasma for routine blood analyses.

\section{In vivo MRI studies}

MRI was captured in live subjects using a 0.5 T MRI system (MiniMR-60; Niumag). For the biodistribution study, GA-Fe@BSA NP mixtures $(150 \mu \mathrm{L}, 20 \mathrm{mM})$ were administered to pathogen-free $\mathrm{BALB} / \mathrm{c}$ nude mice 5-6 weeks old (Shanghai Laboratory Animal Center). Coronal scans were taken before and after NP injections (0.16, 2, 6, and 24 hours). For the tumor MRI study, GA-Fe@BSA NP mixtures $(20 \mu \mathrm{L}$, $23 \mathrm{mM}$ ) were directly injected intratumorally into mammary carcinoma (4T1) tumor-bearing nude mice (Shanghai Institutes for Biological Sciences). Cross-sectional MRI scanned images were taken before and 1 hour after GA-Fe@BSA NP intratumoral injections.

\section{In vivo photothermal ablation of cancer cells}

Mammary carcinoma-tumor models were created by injecting 4T1 cells (Shanghai Institutes for Biological Sciences) into the right hind legs of BALB/c nude mice (5-6 weeks old, Shanghai Laboratory Animal Center). Tumor-bearing $(0.5 \mathrm{~cm})$ mice were randomly assigned to one of four groups (PBS alone, GA-Fe@BSA NPs alone, PBS + laser, and GA-Fe@BSA NPs + laser), with four tumor-bearing $(0.5 \mathrm{~cm})$ mice in each group. For the PBS and PBS + laser group, mice were injected intratumorally with PBS $(20 \mu \mathrm{L})$. In the GA-Fe@BSA NPs and GA-Fe@BSA NPs + laser group, mice were intratumorally injected with GA-Fe@BSA NPs $(20 \mu \mathrm{L}, 15 \mathrm{mM})$. After a 2-hour incubation, the mice in PBS + laser and GA-Fe@BSA NPs + laser group were exposed to an $808 \mathrm{~nm}$ laser at $1 \mathrm{~W} / \mathrm{cm}^{2}$ for 5 minutes. NIR thermal images from the groups receiving laser treatment were taken at time intervals after laser exposure. After photothermal therapy, one subject was killed and tumors evaluated for histopathology by H\&E staining and for apoptosis rates with TUNEL stains. The tumor sizes of the rest of the subjects were recorded every day for 16 days.

\section{Results and discussion Synthesis and characterization of GA-Fe@BSA NPs}

BSA, a low-cost and easily prepared protein, was investigated as a biomineralization agent to prepare metal-organic coordination polymer NPs. The GA-Fe complex was used as biocompatible metal-organic NPs by forming coordination bonds of phenoxide carboxylate groups and $\mathrm{Fe}^{3+}$. $\mathrm{GA}$ is a powerful antioxidant, and $\mathrm{Fe}^{3+}$ is a paramagnetic ion that is less toxic than $\mathrm{Gd}^{3+}$ and $\mathrm{Mn}^{2+} .31,33$ The synthesis method is illustrated in Scheme 1 and Figure S1. First, BSA was dissolved in water. Then, $\mathrm{Fe}^{3+}$ was added to the BSA solution with agitation and the solution became yellow. Given that many amino-acid residues are components of BSA, BSA-Fe complexes were easily formed. In an FT-IR spectrum analysis, the characteristic peak signifying a stretching $\mathrm{Fe}-\mathrm{O}$ bond appeared at $612 \mathrm{~cm}^{-1}$, suggesting the formation of the BSA-Fe complex (Figure S2). In addition, both broadband-enhanced absorption at $280 \mathrm{~nm}$ (Figure S3) and quenched fluorescence (Figure S4) demonstrated the formation of BSA-Fe complexes. ${ }^{34,35}$ Lastly, GA solution was added to the BSA-Fe complex solution at room temperature, with vigorous stirring. The color of the solution changed to purple immediately, which meant the formation of the GA-Fe complex. Circular dichroism spectroscopy (Figure S5) was used to trace conformational changes in the BSA. Results demonstrated that there were no obvious deformations of the BSA during the synthesis process. The synthesized GA-Fe@BSA NPs were incubated for 30 minutes and then collected by ultrafiltration centrifugation, as described earlier.

Synthesized GA-Fe@BSA NPs were first characterized by atomic force microscopy. GA-Fe@BSA NPs were monodispersed, spherical, and with an average diameter of $3.5 \mathrm{~nm}$ (Figure 1). The size of these NPs is thought to be beneficial for biological applications, given that ultrasmall NPs are easily excreted from the human body. ${ }^{7}$ Compared with previous data (in which the average NP diameter was 


\section{A}
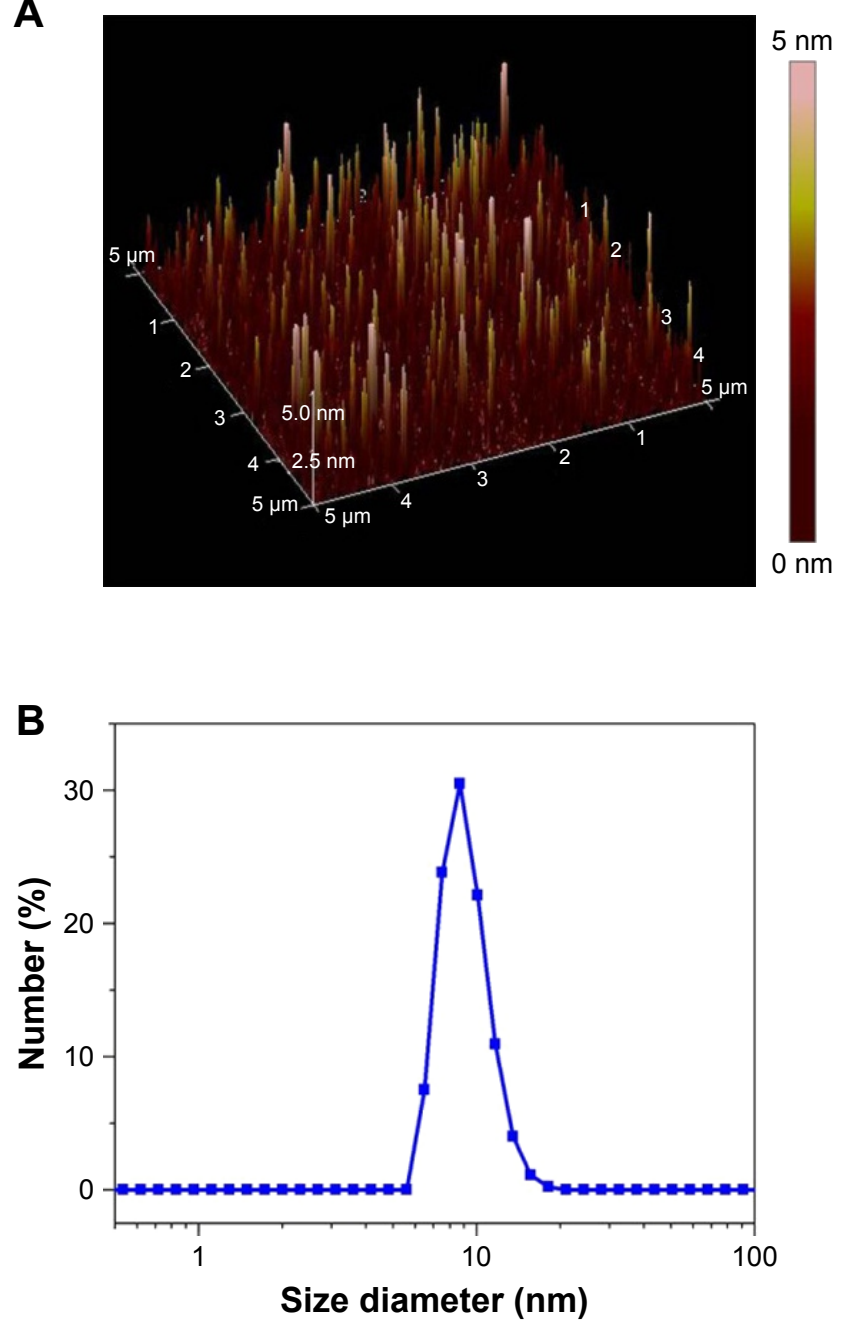

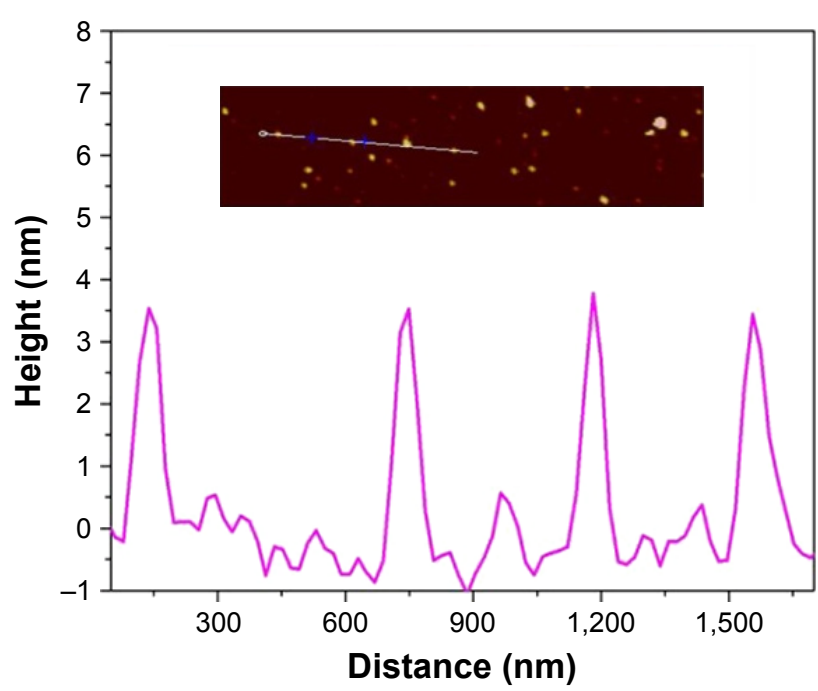

C
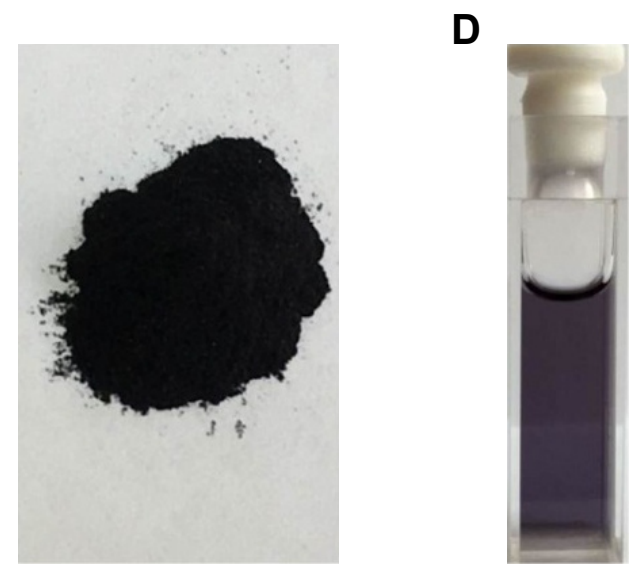

Figure I Characterization of GA-Fe@BSA NPs.

Notes: (A) 3-D AFM topography and height profile (measurements along a line are given after AFM imaging); (B) hydrodynamic size; (C) powder prepared in one synthesis reaction; (D) aqueous solution of GA-Fe@BSA NPs.

Abbreviations: GA, gallic acid; NPs, nanoparticles; AFM, atomic force microscopy.

$42.6 \mathrm{~nm}$ ), the ultrasmall dimensions achieved here can be ascribed to strong coordinating effects between $\mathrm{Fe}^{3+}$ and BSA. ${ }^{28,32}$ Using dynamic light-scattering measurements (Figures 1B and S6), the mean hydrodynamic diameter of GA-Fe@BSA NPs was 8.8 nm, which was slightly larger than results observed with atomic force microscopy. This phenomenon has been explained in a previous study in which the hydrodynamic diameter readings of BSA-coated NPs were slightly larger due to the strong hydrophilicity of BSA. ${ }^{28}$ Importantly, preparation methods for GA-Fe@ BSA NPs can be easily amplified. The yield of synthesized GA-Fe@BSA NPs was nearly 1 g per reaction (Figure 1C). Given that BSA was anchored on GA particles (Figure S7), synthesized GA-Fe@BSA NPs were highly stable in water and dispersed easily, even after 1 month of storage (Figure 1D).
Structural features of the NPs were characterized by measuring FT-IR and UV-visible NIR spectra. FT-IR spectra of GA, BSA, and GA-Fe@BSA NPs are shown in Figure 2A. Characteristic peaks were observed for BSA: $3,301 \mathrm{~cm}^{-1}$ (O-H or $\mathrm{N}-\mathrm{H}$ stretch), $2,965 \mathrm{~cm}^{-1}$ (- $\mathrm{CH}_{2}$ - symmetrical vibrations), $2,870 \mathrm{~cm}^{-1}$ (- $\mathrm{CH}_{2}-$ asymmetric vibrations), $1,650 \mathrm{~cm}^{-1}$ (amide I, mainly $\mathrm{C}=\mathrm{O}$ stretching vibrations), $1,521 \mathrm{~cm}^{-1}$ (amide II, coupling of bending vibrations of $\mathrm{N}-\mathrm{H}$ and stretching vibrations of $\mathrm{C}-\mathrm{N}$ ), $1,391 \mathrm{~cm}^{-1}$ (side chain $\mathrm{COO}^{-}$), and $1,241 \mathrm{~cm}^{-1}$ (C-N stretching/N-H bending vibrations). ${ }^{36}$ Similarly, GA characteristic peaks were observed: $3,376 \mathrm{~cm}^{-1}$ (O-H stretch), 3,290 $\mathrm{cm}^{-1}$ (O-H stretch), 1,706 $\mathrm{cm}^{-1}$ $(\mathrm{C}=\mathrm{O}$ stretch $), 1,616 \mathrm{~cm}^{-1}$ (C-C stretch), 1,544 $\mathrm{cm}^{-1}(\mathrm{C}-\mathrm{C}$ stretch), $1,442 \mathrm{~cm}^{-1}$ (C-O stretch), 1,344 $\mathrm{cm}^{-1}$ (C-C stretch), $1,254 \mathrm{~cm}^{-1}$ (C-O stretch), $1,031 \mathrm{~cm}^{-1}$ (O-H in-plane deformation), $866 \mathrm{~cm}^{-1}$ (C-H out-of-plane bending), and $704 \mathrm{~cm}^{-1}$ 

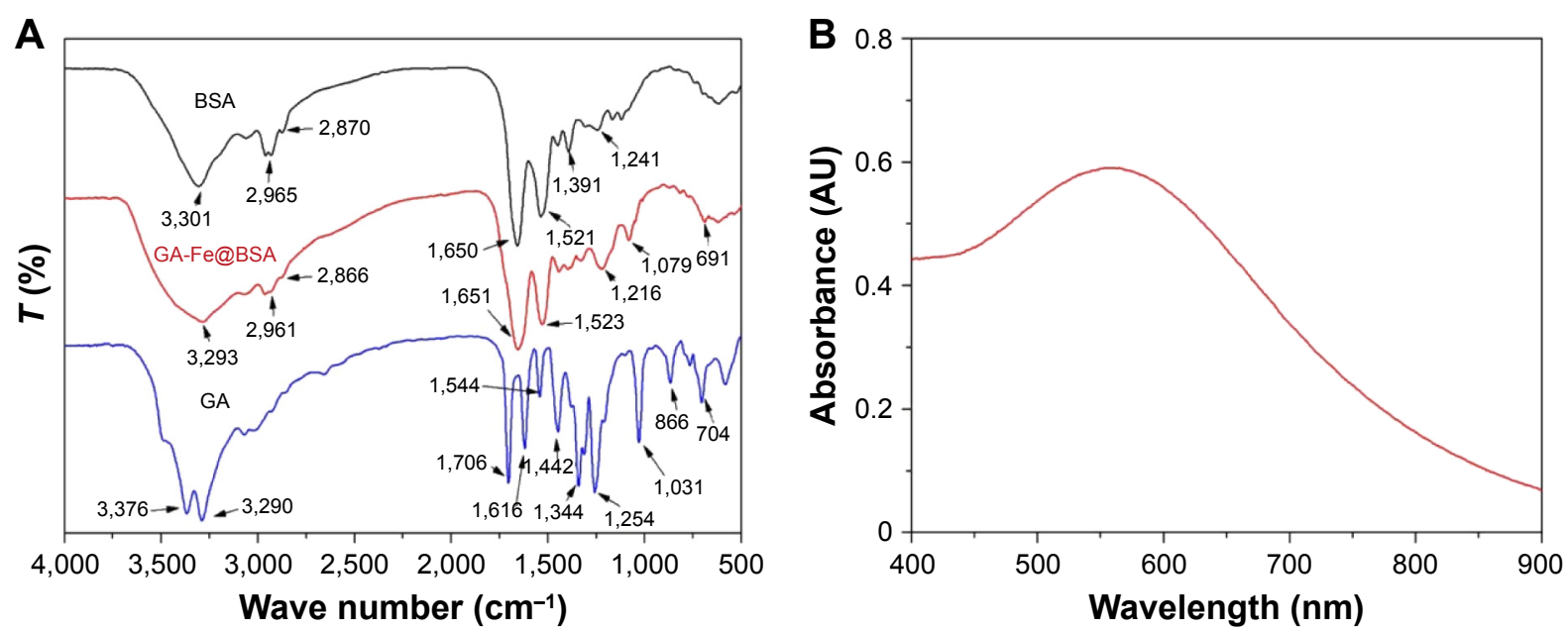

Figure 2 FT-IR (A) and UV-visible NIR absorption spectra (B) of GA-Fe@BSA NPs. Abbreviations: FT-IR, Fourier-transform infrared; UV, ultraviolet; NIR, near-IR; GA, gallic acid; NPs, nanoparticles.

(C-H out-of-plane bending). ${ }^{37}$ GA-Fe@BSA NPs possessed similar peaks that were characteristic of BSA - amide I band vibrations at $1,650 \mathrm{~cm}^{-1}$ and amide II band vibrations at $1,521 \mathrm{~cm}^{-1}$ - supporting the presence of BSA in the GA-Fe@ BSA NPs. The small shift in the amide I band vibration and quenched fluorescence of the GA-Fe@BSA NPs suggested that an interaction was occurring between $\mathrm{Fe}^{3+}$ and the amide of the BSA (Figure S4). ${ }^{35}$ Disappearance of O-H stretching at $3,376 \mathrm{~cm}^{-1}$ and $3,290 \mathrm{~cm}^{-1}$, as well as the $\mathrm{O}-\mathrm{H}$ of the carboxylic group bending the band at $1,031 \mathrm{~cm}^{-1}$, indicated that the phenolic groups and carboxylic groups in GA chelated with $\mathrm{Fe}^{3+}$ in GA-Fe@BSA NPs. ${ }^{38}$

UV-visible NIR absorption spectra (Figure 2B) illustrated that GA-Fe@BSA NPs had strong absorption in the entire visible NIR region, with a peak at $558 \mathrm{~nm}$, which might have belonged to $d-d$ electronic transitions of the GA-Fe complex. ${ }^{32}$ Together, these data demonstrate that BSA-tuned GA-Fe NPs can be successfully synthesized.

\section{Photothermal properties of GA-Fe@BSA NPs}

Given the absorption profile observed with GA-Fe@BSA NPs, further investigations were performed to assess photothermal conversion effects. Temperature variations in aqueous GA-Fe@BSA NP mixtures were monitored with an IR thermal-imaging camera, while solutions were exposed to $808 \mathrm{~nm}$ laser irradiation at $1 \mathrm{~W} / \mathrm{cm}^{2}$ for 15 minutes. GA-Fe@ BSA NP concentrations varied from 0 to $1 \mathrm{mM}$. Imaging colors of each solution varied greatly after exposure to laser treatment (Figure 3A), signifying that temperatures elevated quickly with increasing concentrations of GA-Fe@BSA NPs. Qualitatively observed color differences in imaging data were consistent with photothermal measurements (Figure 3B). As GA-Fe@BSA NPs concentrations increased, temperature changes over the 15 -minute time course increased from $3.4^{\circ} \mathrm{C}$ to $25^{\circ} \mathrm{C}$. Pure-water temperatures increased only $1.4^{\circ} \mathrm{C}$ under the same conditions. These results demonstrated that ultrasmall GA-Fe@BSA NPs absorbed laser energy and efficiently converted it to heat. The practical application for this feature is photothermal ablation to treat solid tumors.

Photostability was measured by testing temperature changes in GA-Fe@BSA NP solutions as they were subjected to laser on/off cycles (Figure 3C). UV-visible NIR spectra were measured before and after laser treatments (Figure S8). After eight laser on/off cycles, temperature increments in the GA-Fe@BSA NP aqueous mixtures changed just slightly. Furthermore, the UV-visible NIR spectra of GA-Fe@BSA NP aqueous mixtures exhibited similar peak curves before and after laser exposure. These results suggested that GA-Fe@BSA NPs were photostable.

\section{MRI of GA-Fe@BSA NPs}

Longitudinal $\left(T_{1}\right)$ and transverse $\left(T_{2}\right)$ relaxation times were tested to evaluate whether GA-Fe@BSA NPs could serve as an MRI contrast agent. Relaxivity values of GA-Fe@BSA NPs were $0.89 \mathrm{mM} / \mathrm{second}$ for $r_{1}$ and $0.95 \mathrm{mM} / \mathrm{second}$ for $r_{2}$. These results were comparable with previous reports of other MRI contrast agents. ${ }^{31,39}$ However, the $r_{2}: r_{1}$ ratio of GA-Fe@BSA NPs, calculated from the data presented in Figure 4A $\left(r_{2}: r_{1}=1.06<3\right)$, was relatively lower than previously described agents. These results suggested that GA-Fe@, BSA NPs would provide good $T_{1}$-weighted contrast. The potential application of GA-Fe@BSA NPs as a $T_{1}$-weighted MRI contrast agent was explored (Figure 4B). The subjective 

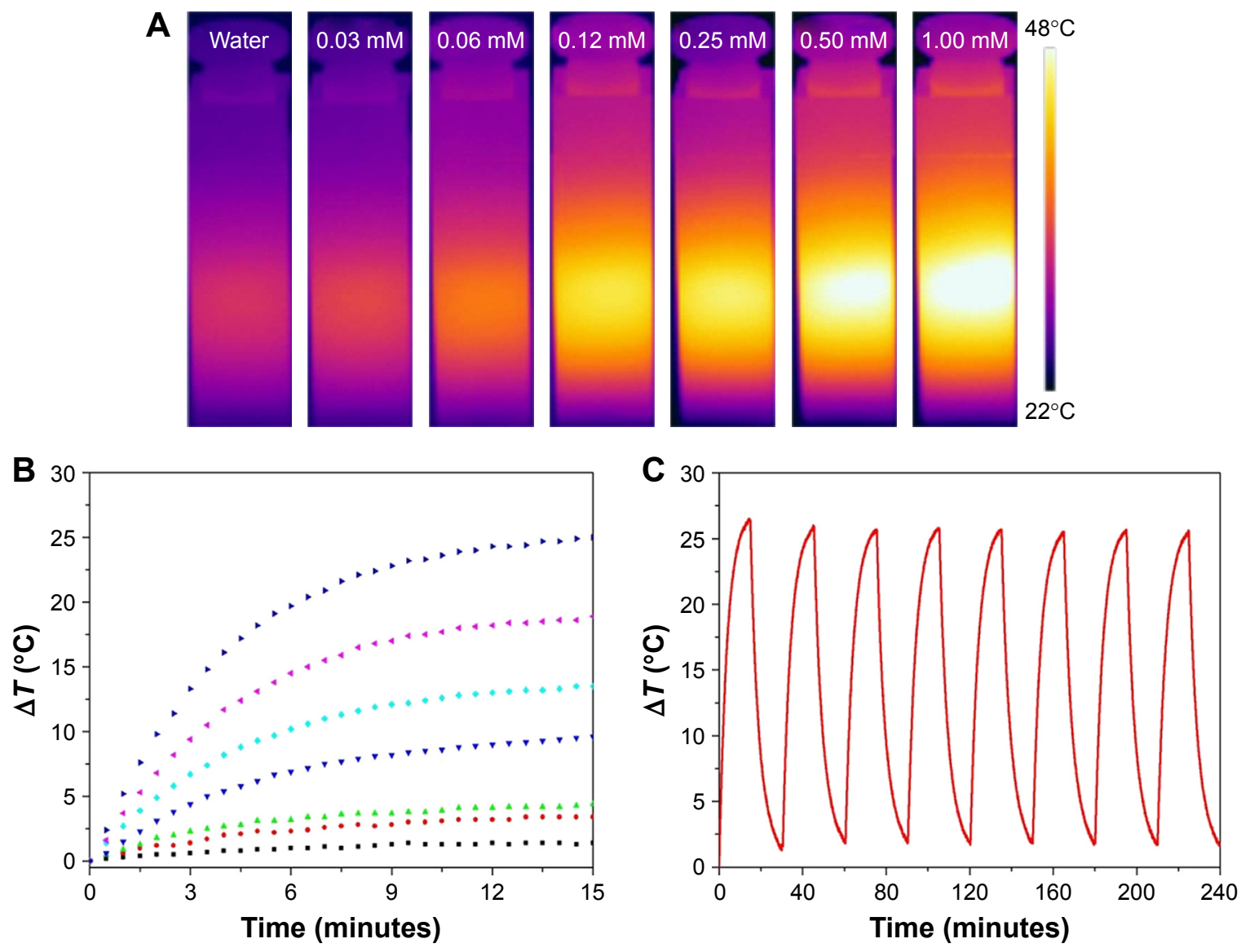

\begin{tabular}{lll}
\hline - Water & $\cdot 0.03 \mathrm{mM}$ & $\triangle 0.06 \mathrm{mM}$ \\
- $0.10 \mathrm{mM}$ & $\cdot 0.25 \mathrm{mM}$ & $\triangleleft 0.50 \mathrm{mM}$ \\
$-1.00 \mathrm{mM}$ & &
\end{tabular}

Figure 3 Photothermal performance of the GA-Fe@BSA NPs.

Notes: (A) NIR thermal images and (B) temperature variations of GA-Fe@BSA NPs at increasing concentrations, after laser ablation in the same conditions. (C) Photostability tests for water dispersion of GA-Fe@BSA NPs after eight laser on/off cycles.

Abbreviations: NIR, near-infrared; GA, gallic acid; NPs, nanoparticles.

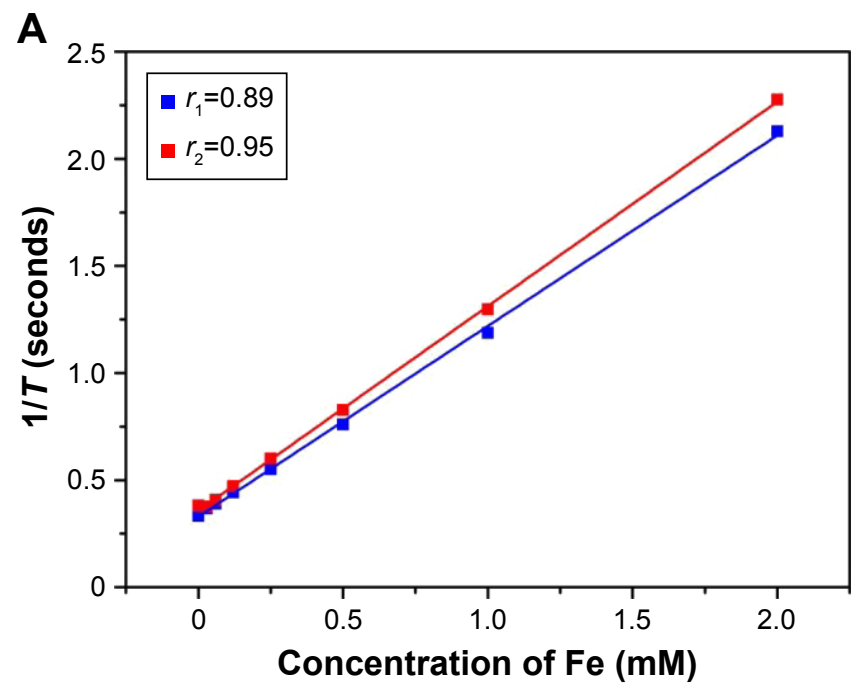

B

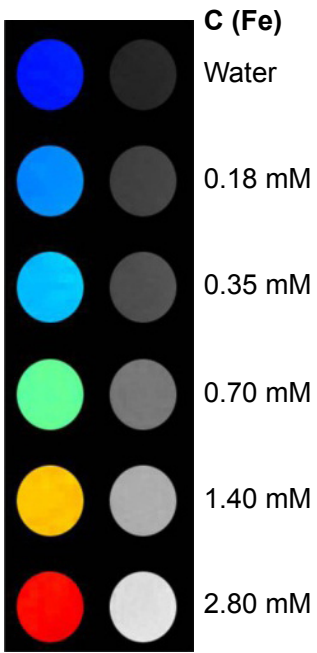

Figure 4 (A) Relaxivity $\left(r_{1}\right.$ and $r_{2}$ ) and (B) $T_{1}$-weighted MRI images of GA-Fe@BSA NPs versus $\mathrm{Fe}^{3+}$ concentrations in solution. Abbreviations: MRI, magnetic resonance imaging; GA, gallic acid; NPs, nanoparticles. 
colors of $T_{1}$-weighted images gradually shifted from dark (pure water) to light with increased concentrations of aqueous GA-Fe@BSA NP mixtures. Given these results, GA-Fe@ BSA NPs had encouraging potential as a $T_{1}$-weighted MRI contrast agent for biomedical diagnostics.

\section{Biocompatibility of GA-Fe@BSA NPs}

The biocompatibility of GA-Fe@BSA NPs was evaluated using cytotoxicity tests on human macrophages, hemolysis assays on RBCs, cell-cycle assays on HEK293T cells, and in vivo toxicity analyses by routine blood testing. Cell viability was tested in human macrophages after 12- or 24-hour incubation with increasing concentrations of GA-Fe@BSA NPs (Figure 5A). Compared with controls, the viability of GA-Fe@BSA NP-treated human macrophages was modestly reduced. Nonetheless, cell viability remained greater than $80 \%$ in samples incubated with GA-Fe@BSA NPs at the highest concentration $(200 \mu \mathrm{M})$ and longest time (24 hours).
A

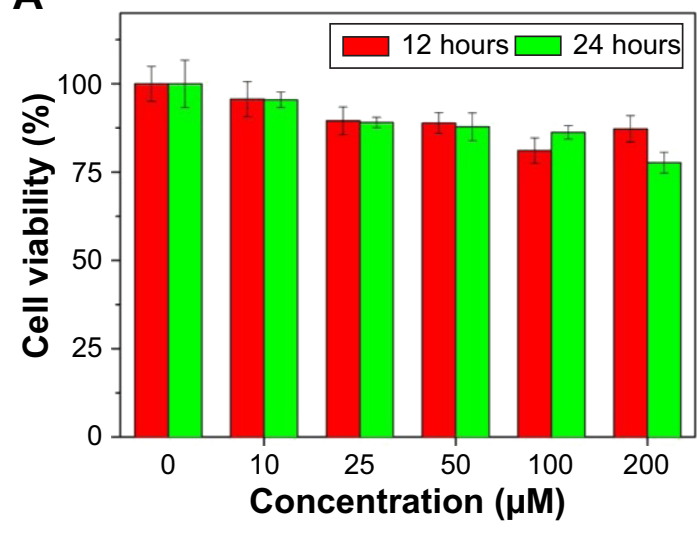

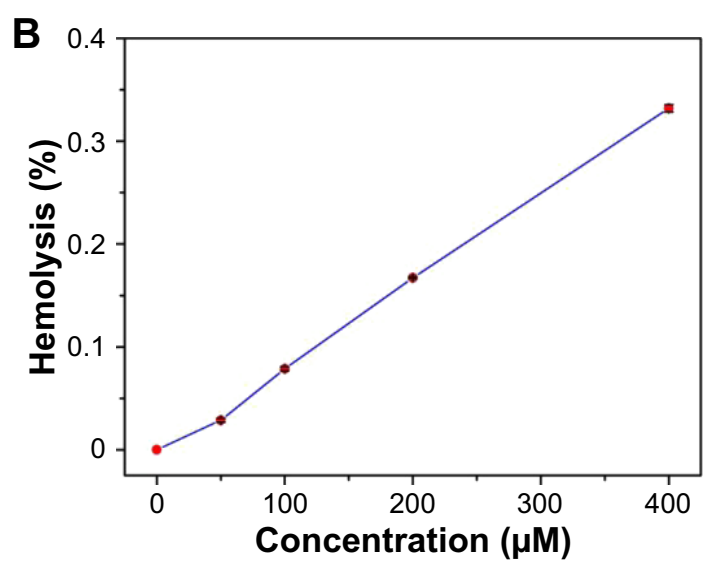

C

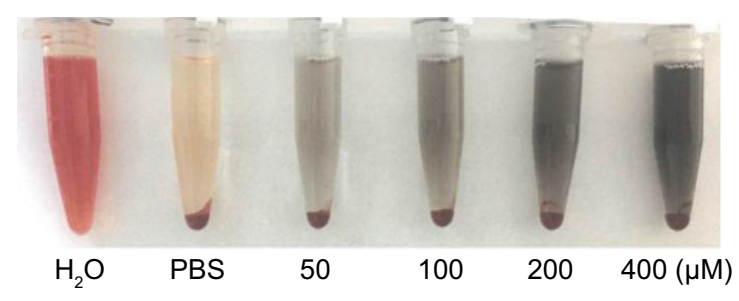

D

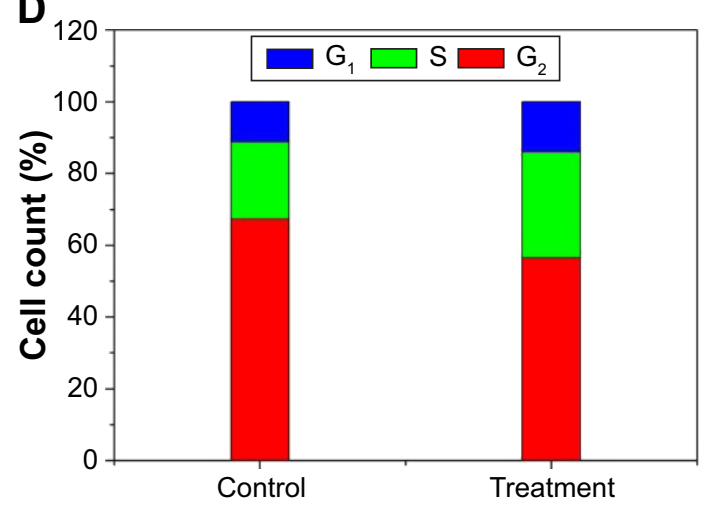

$\mathbf{E}$

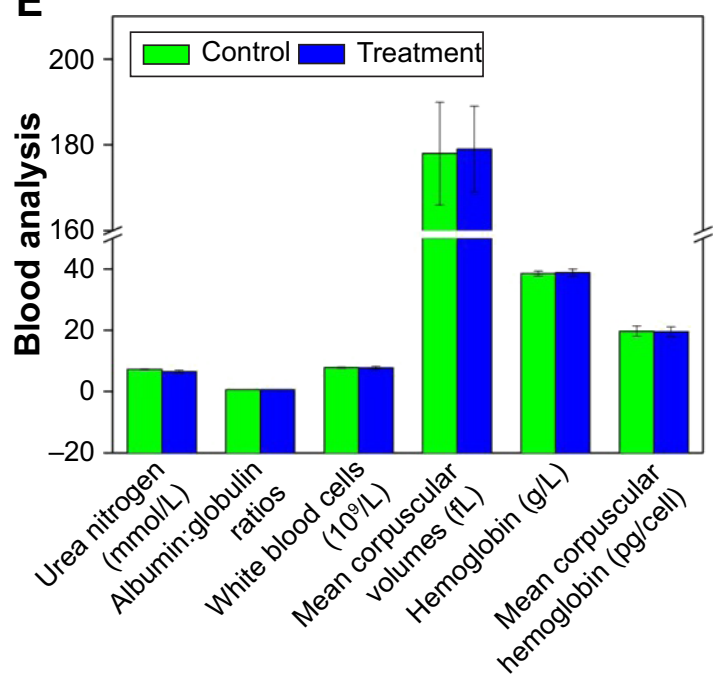

Figure 5 The biocompatibility of GA-Fe@BSA NPs.

Notes: (A) Cell viability of human macrophages after incubation with GA-Fe@BSA NPs at different concentrations (0, 10, 25, 50, 100, and 200 mM) for 12 and 24 hours. (B) Rates of hemolysis in RBCs after I-hour incubations with increasing GA-Fe@BSA NP concentrations. (C) Visual differences observed in RBCs treated with water, PBS, and concentrations of GA-Fe@BSA NPs after centrifugation. (D) Cell-cycle analyses of HEK293T cells treated with $200 \mu$ M GA-Fe@BSA NPs for 24 hours. (E) Routine blood analyses for subjects after intravenous infusion of GA-Fe@BSA NPs (200 $\mu$ L, 20 mM for each mouse) for 3 days: urea nitrogen levels, albumin:globulin ratios, white blood-cell counts, hemoglobin levels, mean corpuscular volumes, and mean corpuscular hemoglobin levels.

Abbreviations: GA, gallic acid; NPs, nanoparticles; RBCs, red blood cells. 
Therefore, GA-Fe@BSA NPs exhibited low cytotoxicity $(>80 \%)$ overall when used at concentrations $<200 \mu \mathrm{M}$.

Next, hemolysis assays were performed on mouse erythrocytes. The hemolysis ratio of GA-Fe@BSA NPs for all tested concentrations was lower than $0.33 \%$ (Figure $5 \mathrm{~B}$; much lower than the permissible limit of 5\%). Therefore, no gross hemolysis was observed with GA-Fe@BSA NP treatment at concentrations $<400 \mu \mathrm{M}$. Furthermore, RBCs treated with GA-Fe@BSA NPs at $400 \mu \mathrm{M}$ concentrations had similar hemolytic responses to the negative controls (Figure 5C).

Cell cycles of HEK293T cells were measured. Results suggested that GA-Fe@BSA NPs did not significantly impact cell-cycle distributions in comparison with controls (Figure 5D). The results of cytokinesis-blocked micronucleus assays also further demonstrated that GA-Fe@BSA NPs exhibited no noteworthy genotoxicity (Figure S9).

Additionally, routine blood analyses were performed to evaluate toxicity in mouse models: urea nitrogen, albumin:globulin ratios, white blood-cell counts, hemoglobin, mean corpuscular volume, and mean corpuscular hemoglobin. All test results were normal in subjects treated with GA-Fe@BSA NPs at $200 \mu \mathrm{L}$ concentrations when compared with control subjects. Taken together, these results suggested that GA-Fe@BSA NPs exhibited good biocompatibility.

\section{In vivo biodistribution and clearance of GA-Fe@BSA}

For human use of in vivo infused agents, the FDA requires that they be excreted from the body over a reasonable time course. ${ }^{13}$ As such, ultrasmall GA-Fe@BSA NPs were tested for body-clearance rates. The biodistribution of GA-Fe@ BSA NPs in healthy mice was followed by tracking changes in MR signals after application of the reagent. $T_{1}$-weighted MRI of murine subjects was recorded prior to and at 0.16 , 2, 6, and 24 hours after intravenous infusion of GA-Fe@ BSA NPs (Figure 6). $T_{1}$-weighted MRI of the mice before and 0.16 hours after infusion was compared. At this time point, MR signals accumulated in the kidneys and bladder; the signal in the liver was relatively weak. Decay of MR signals began at 2 and 6 hours after infusion for the kidneys and liver, respectively. The MR signal in the bladder continued to increase at 6 hours after administration. At 24 hours posttreatment, MR signals returned to basal levels observed in all organs prior to treatment. To be noted, extensive MR signals observed in the kidneys and bladder revealed that ultrasmall GA-Fe@BSA NPs were excreted from the body through renal filtration pathways. Moreover, relatively small changes in MR signals observed in the liver suggested that only a small amount of GA-Fe@BSA NPs was intercepted by the reticuloendothelial system.

In order to identify if the GA-Fe@BSA NPs were degraded in vivo, the urine and blood of the mice administered PBS and GA-Fe@BSA NPs at 0 and 24 hours were collected. After ultrafiltration centrifugation with molecular weight cutoff of $3 \mathrm{kD}$, iron concentration in the urine and blood was acquired by inductively coupled plasma. Slight differences in urine and blood iron concentrations between subjects administered PBS versus GA-Fe@BSA NPs were observed at 0 and 24 hours (Figure S10). This suggested that GA-Fe@BSA NPs were stable in vivo and easily excreted from the body. Altogether, these results demonstrate that ultrasmall GA-Fe@BSA NPs can effectively avoid phagocytosis by the reticuloendothelial system and are easily eliminated through renal filtration systems.

\section{In vivo tumor $M R I$}

Imaging is a critical function for a novel nanotheranostic agent; these classes of agents are typically employed in detection and evaluation of cancer therapeutic effects. ${ }^{3}$ Here, GA-Fe@BSA NP MRI properties were evaluated in nude mice bearing 4T1 breast tumors. As shown in Figure 7A, marked positive accumulation of GA-Fe@BSA NPs was observed in $T_{1}$-weighted MRI of 4T1 breast tumors acquired 1 hour after intratumoral injections with reagents. Signal intensity of tumors 1 hour after intratumoral injection was nearly twice as high as preinjection intensity (Figure 7B). These results demonstrated that GA-Fe@BSA NPs were successfully applied as $T_{1}$-weighted MRI contrast agents that captured tumor-size differences occurring in vivo.

\section{In vivo photothermal therapy}

NIR thermal imaging, a method that quantifies spatial temperature distribution, allows real-time evaluation of thermal treatment effects on tumors. ${ }^{4}$ NIR thermal images were recorded of 4T1 tumor-bearing subjects who were treated with intratumoral GA-Fe@BSA NP injections, with increased lengths of laser ablation (Figure 8A). While being ablated, the rate of temperature rise in GA-Fe@BSA NPsinjected subjects was faster than controls. Also increments of temperature increase were higher than controls (Figure 8B), suggesting that better in vivo photothermal therapy was occurring when GA-Fe@BSA NPs and laser technologies were combined. Subsequent histological examinations assessed photothermal treatment effects on tumor tissues. Representative images of tumor sections taken from each of the four groups are given (Figure 8C). In these experiments, tumor specimens were harvested 2 hours after intratumoral 

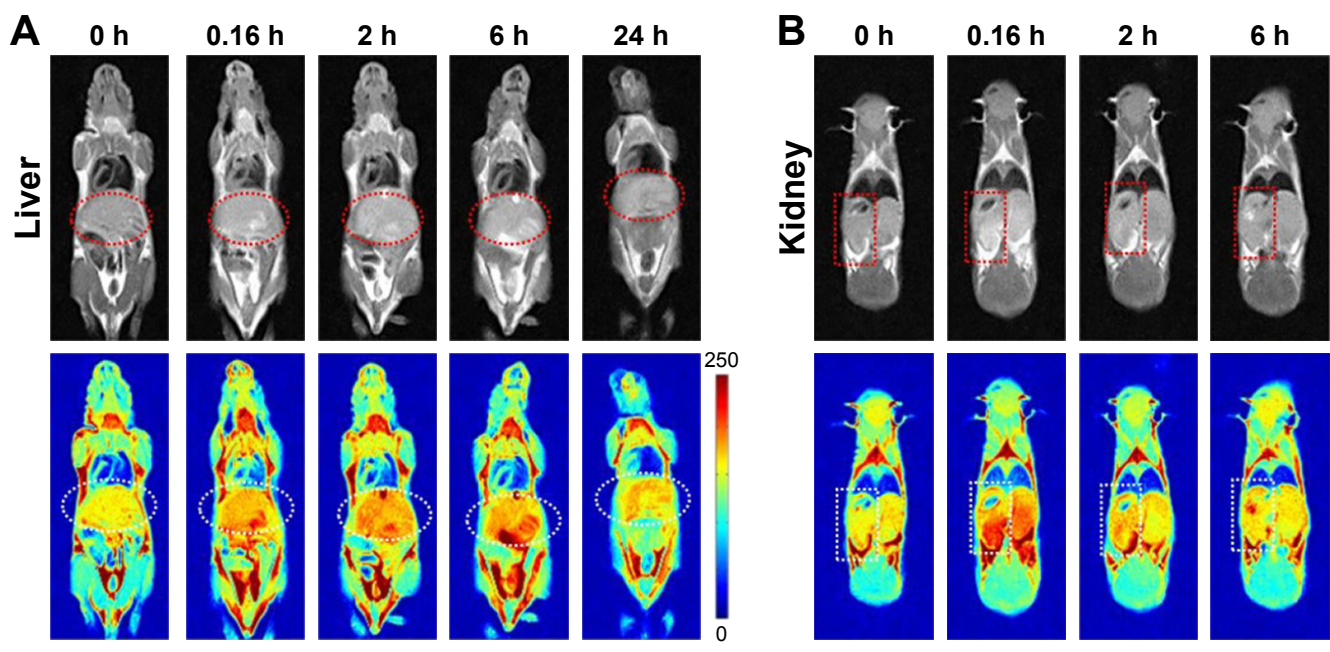

$24 \mathrm{~h}$
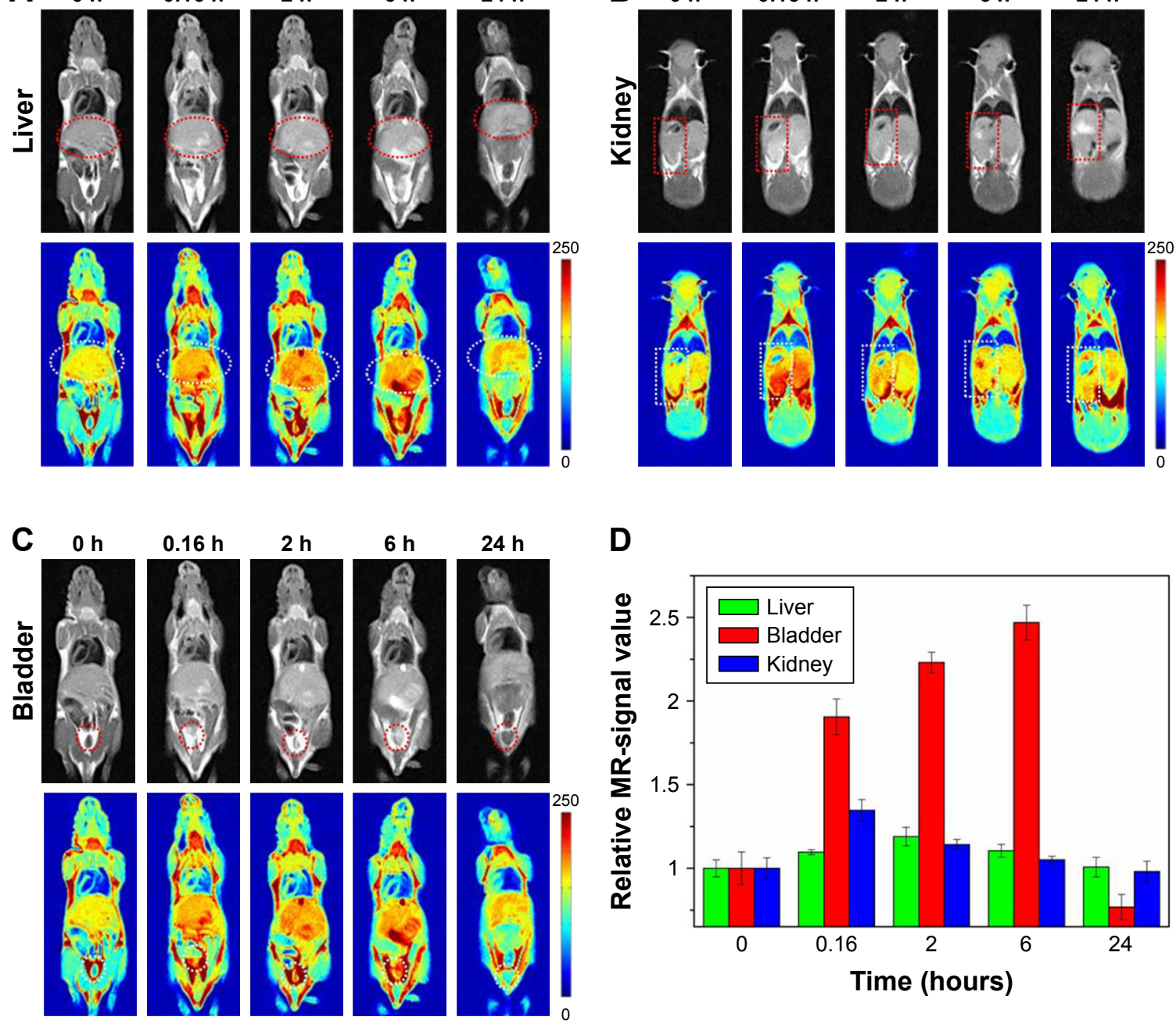

D

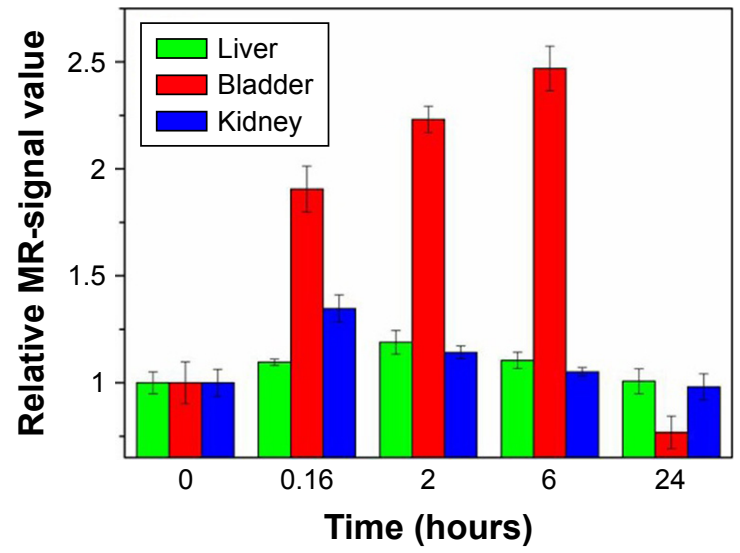

Figure 6 MRI findings.

Notes: In vivo images of $(\mathbf{A})$ livers (marked with ellipses), (B) kidneys (marked with rectangles), and (C) bladders (marked with circles) of subjects before (0 h) and after $(0.16,2,6$, and $24 \mathrm{~h}$ ) intravenous infusion with GA-Fe@BSA NPs. (D) Corresponding MR-signal intensities of MRI shown in (A-C).

Abbreviations: MRI, magnetic resonance imaging; GA, gallic acid; NPs, nanoparticles.
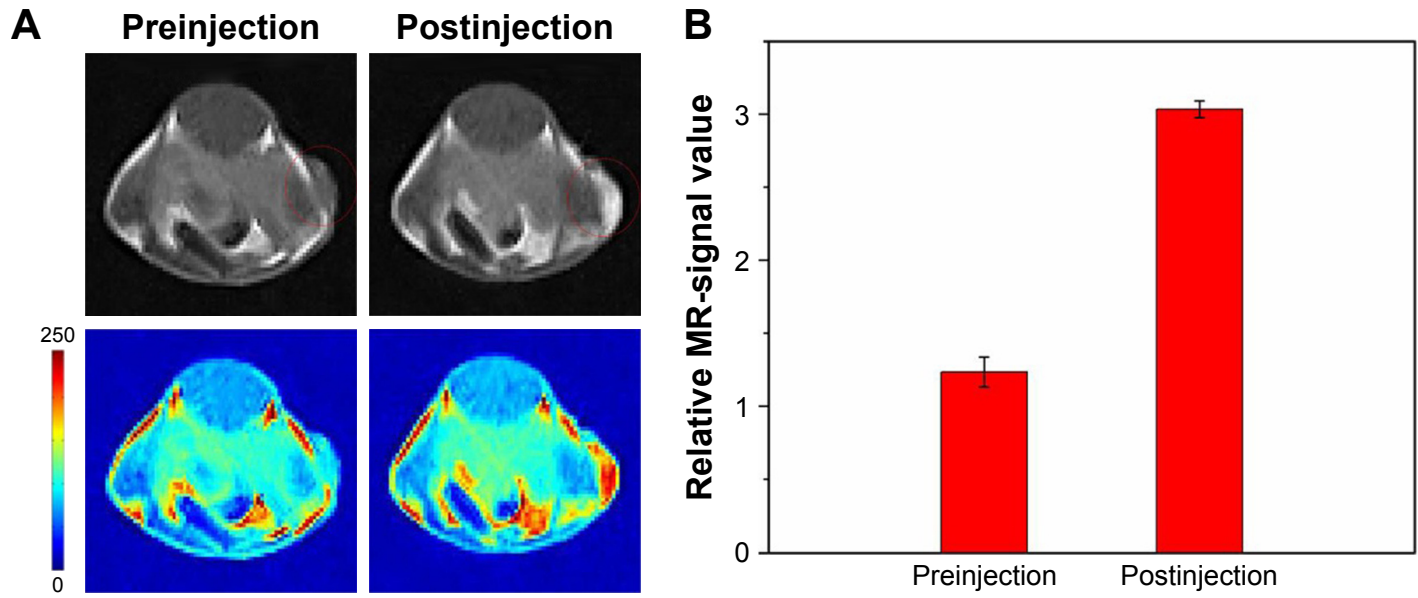

Figure 7 (A) MRI and (B) corresponding relative signal values of tumor-bearing subjects before and after intratumoral injection of GA-Fe@BSA NPs. Abbreviations: MRI, magnetic resonance imaging; GA, gallic acid; NPs, nanoparticles. 
A

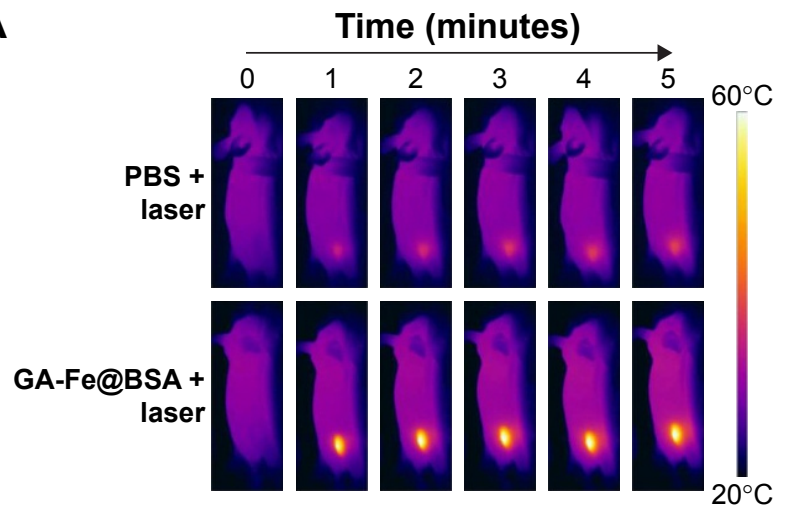

B

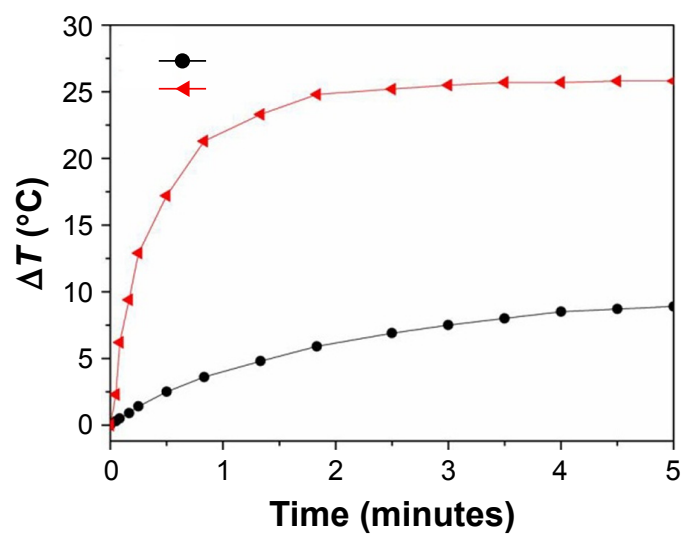

C

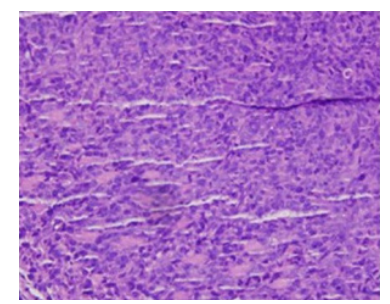

GA-Fe@BSA

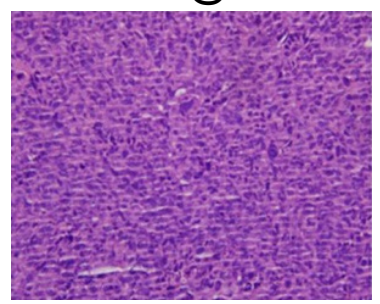

PBS + laser

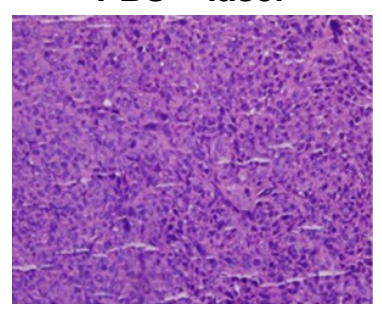

GA-Fe@BSA + laser

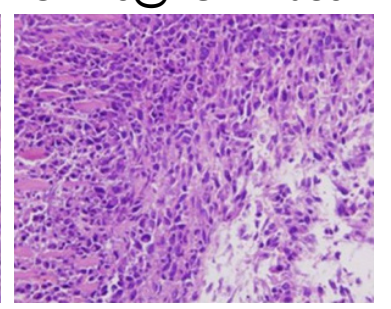

Figure 8 In vivo photothermal therapy.

Notes: NIR thermal images (A) and corresponding temperature variations (B) of mice bearing tumors after intratumoral injection with PBS or GA-Fe@BSA and receiving laser ablation; (C) representative images of tumor sections taken from each exposure group (magnification 40×).

Abbreviations: NIR, near-infrared; GA, gallic acid.

injection with PBS or GA-Fe@BSA NPs, with or without laser-ablation treatment $\left(1 \mathrm{~W} / \mathrm{cm}^{2}, 5\right.$ minutes $)$. For the group treated with GA-Fe@BSA NPs and laser therapies, tumor sections exhibited visible differences from all other conditions. For instance, cell densities appeared low in these subjects. The cells also possessed obvious thermonecrotic features (ie, cell shrinkage, coagulation, and nuclear damage). Differences were also observed with TUNEL staining (Figure S11). The GA-Fe@BSA NPs and laser ablationtreated group showed extensive regions stained brown, denoting apoptotic cells. Taken together, these experiments demonstrated that GA-Fe@BSA NPs coupled with laser ablation destroyed solid-tumor tissue in vivo.

To evaluate further the photothermal therapeutic effects of GA-Fe@BSA NPs, changes to tumors were investigated after various treatments. Murine subjects were randomized to one of four groups treated with PBS, GA-Fe@BSA NPs, PBS plus laser ablation, or GA-Fe@BSA NPs plus laser ablation. The tumor-treatment group receiving GA-Fe@BSA NPs and laser ablation exhibited an incrustation 8 days after treatment. This was lost by the 16-day posttreatment time point (Figure 9A). The other three groups had similar changes in peritumoral edema at 8 days posttreatment. Necrotic regions in the tumor appeared at 16 days after treatment, owing to rapid growth of the tumor. Tumor size, as measured daily with calipers, was notably reduced in subjects receiving both GA-Fe@BSA NPs and laser ablation (Figure 9B). Slower tumor growth was observed in the PBS group receiving laser ablation. Faster tumor growth was observed in mice receiving any treatment without laser ablation. Photographs of dissected tumors, gathered 16 days after treatment, further supported that the GA-Fe@BSA NPs plus laser group exhibited complete tumor ablation (Figure 9C). These results suggested that higher temperatures (approximately $52^{\circ} \mathrm{C}$ ) generated by GA-Fe@BSA NPs after laser ablation had antitumor activities. Subjects treated with PBS or GA-Fe@, BSA NPs without laser ablation had lower temperatures and less antitumor activity; temperatures stimulated by PBS plus laser also produced a limited range of inhibitory effects on tumor growth.

\section{Conclusion}

In summary, BSA was investigated as a substrate used to control the synthesis of GA-Fe(III) coordination polymer NPs. 

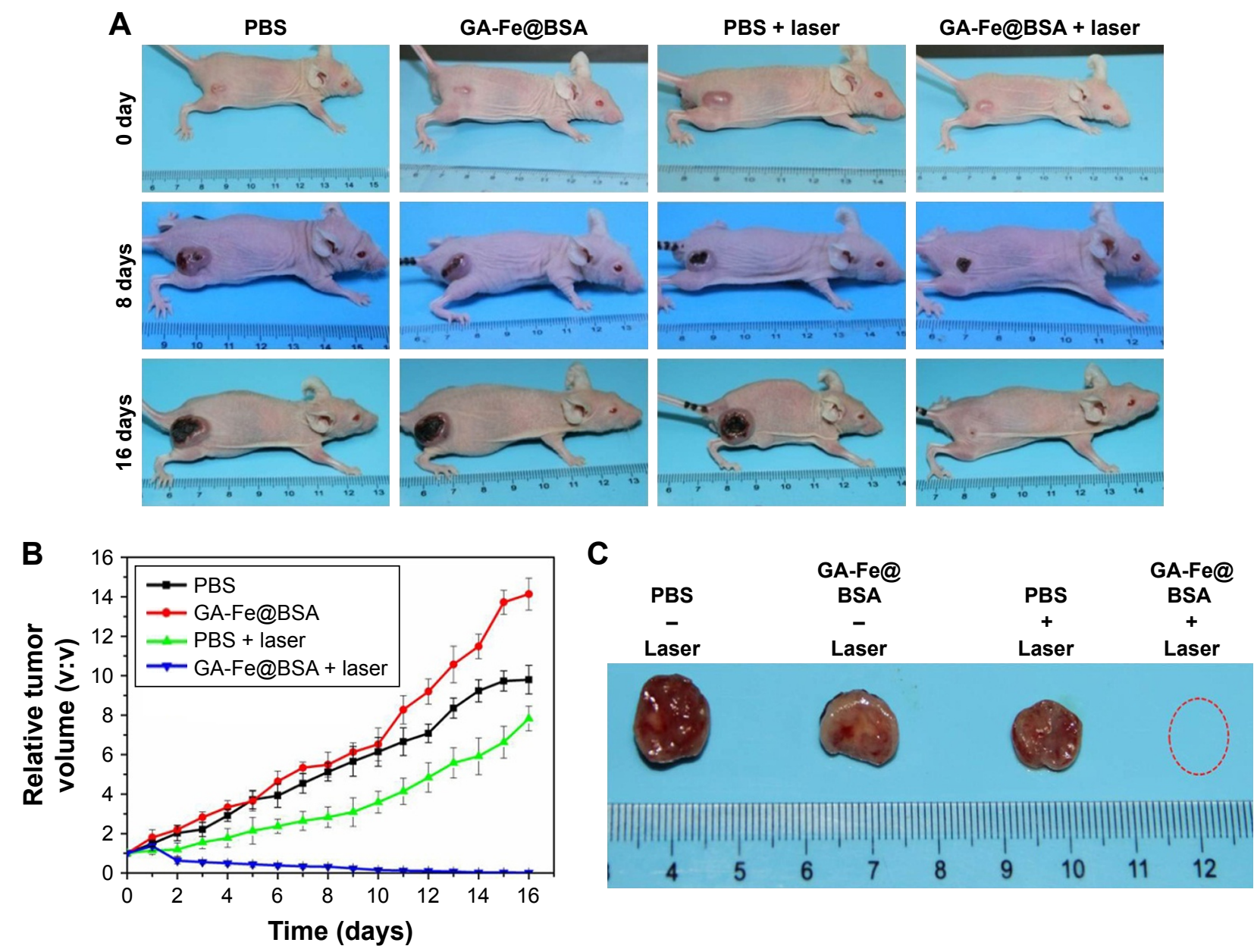

Figure 9 Tumor changes after photothermal therapy.

Notes: (A) Representative images and (B) profiles of tumor volumes in mice, with different treatments at different time points; (C) representative images of dissected tumors from mice 16 days after different treatments without $(-)$ or with laser ablation $(+)$. The red circle denotes that no tumor was dissected.

Abbreviation: GA, gallic acid.

GA-Fe@BSA NPs were ultrasmall (3.5 nm) due to strong interactions between BSA and $\mathrm{Fe}^{3+}$. These particles showed good biocompatibility, and a lower $r_{2}: r_{1}$ ratio (1.06). Strong absorption in the visible NIR region of GA-Fe@BSA NPs gave them the potential application as an MRI diagnosis and photothermal therapy tool. Moreover, ultrasmall GA-Fe@ BSA NPs effectively avoided phagocytosis in the reticuloendothelial system and were easily eliminated through renal filtration. This quality also reduced potential toxicity induced by long-term corporal retention in the injected agents. Remarkable signal changes in $T_{1}$-weighted MRI were observed in tumor-bearing subjects before and after intratumor injection with GA-Fe@BSA, which efficiently destroyed solid tumors that were also treated with laser ablation. Together, these data suggest a bright future for GA-Fe@BSA NPs as safe and efficient theranostic agents for use in clinical applications. The extensive experiments shown here demonstrated that high-performance GA-Fe@
BSA NPs could help in the development and improvement of current diagnostic nanoplatforms.

\section{Acknowledgments}

This work was partially supported by the National Natural Science Foundation of China (21601124 and 21671135), Program of Young Eastern Scholar from Shanghai Institutions of Higher Learning (QD2015038), Shanghai Rising Star Program (17QA1402600), Natural Science Foundation of Shanghai (16ZR1424700), Ministry of Education of China (PCSIRT_IRT_16R49), and the International Joint Laboratory on Resource Chemistry (IJLRC).

\section{Disclosure}

The authors report no conflicts of interest in this work.

\section{References}

1. Liu JN, Bu WB, Shi JL. Chemical design and synthesis of functionalized probes for imaging and treating tumor hypoxia. Chem Rev. 2017;117(9): 6160-6622. 
2. Huynh E, Leung BY, Helfield BL, et al. In situ conversion of porphyrin microbubbles to nanoparticles for multimodality imaging. Nat Nanotechnol. 2015;10(4):325-332.

3. Patra M, Zarschler K, Pietzsch HJ, Stephan H, Gasser G. New insights into the pretargeting approach to image and treat tumours. Chem Soc Rev. 2016;45(23):6415-6431.

4. Cheng L, Wang C, Feng LZ, Yang K, Liu Z. Functional nanomaterials for phototherapies of cancer. Chem Rev. 2014;114(21):10869-10939.

5. Huang XH, El-Sayed IH, Qian W, El-Sayed MA. Cancer cell imaging and photothermal therapy in the near-infrared region by using gold nanorods. J Am Chem Soc. 2006;128(6):2115-2120.

6. Song JB, Yang XY, Jacobson O, et al. Ultrasmall gold nanorod vesicles with enhanced tumor accumulation and fast excretion from the body for cancer therapy. Adv Mater. 2015;27(33):4910-4917.

7. Zhou M, Li JJ, Liang S, Sood AK, Liang D, Li C. CuS nanodots with ultrahigh efficient renal clearance for positron emission tomography imaging and image-guided photothermal therapy. ACS Nano. 2015;9(7): 7085-7096.

8. Wen L, Chen L, Zheng SM, et al. Ultrasmall biocompatible $\mathrm{WO}_{3-x}$ nanodots for multi-modality imaging and combined therapy of cancers Adv Mater. 2016;28(25):5072-5079.

9. Zhang YX, Zhang H, Wang YK, et al. Hydrophilic graphene oxide/ bismuth selenide nanocomposites for CT imaging, photoacoustic imaging, and photothermal therapy. J Mater Chem B Mater Biol Med 2017;5(9):1846-1855.

10. Sun BM, Wu JR, Cui SB, et al. In situ synthesis of graphene oxide/gold nanorods theranostic hybrids for efficient tumor computed tomography imaging and photothermal therapy. Nano Res. 2017;10(1):37-48.

11. Sobhani Z, Behnam MA, Emami F, Dehghanian A, Jamhiri I. Photothermal therapy of melanoma tumor using multiwalled carbon nanotubes. Int J Nanomedicine. 2017;12:4509-4517.

12. Yang $\mathrm{K}, \mathrm{Xu} \mathrm{H}$, Cheng L, Sun CY, Wang J, Liu Z. In vitro and in vivo near-infrared photothermal therapy of cancer using polypyrrole organic nanoparticles. Adv Mater. 2012;24(41):5586-5592.

13. Liu JB, Yu MX, Zhou C, Zheng J. Renal clearable inorganic nanoparticles: a new frontier of bionanotechnology. Mater Today. 2013; 16(12):477-486

14. Taratula O, Schumann C, Naleway MA, Pang AJ, Chon KJ, Taratula O. A multifunctional theranostic platform based on phthalocyanine-loaded dendrimer for image-guided drug delivery and photodynamic therapy. Mol Pharm. 2013;10(10):3946-3958.

15. Li YP, Lin TY, Luo Y, et al. A smart and versatile theranostic nanomedicine platform based on nanoporphyrin. Nat Commun. 2014;5:4712.

16. Sun Y, Du XQ, He JL, Hu J, Zhang MZ, Ni PH. Dual-responsive core-crosslinked polyphosphoester-based nanoparticles for $\mathrm{pH} / \mathrm{redox}$ triggered anticancer drug delivery. J Mater Chem B Mater Biol Med. 2017;5(20):3771-3782.

17. Song XJ, Chen Q, Liu Z. Recent advances in the development of organic photothermal nano-agents. Nano Res. 2015;8(2):340-354.

18. Huang P, Rong PF, Jin A, et al. Dye-loaded ferritin nanocages for multimodal imaging and photothermal therapy. Adv Mater. 2014;26(37): 6401-6408.

19. Rong PF, Huang P, Liu ZG, et al. Protein-based photothermal theranostics for imaging-guided cancer therapy. Nanoscale. 2015;7(39): 16330-16336.

20. Todd TJ, Zhen ZP, Xie J. Ferritin nanocages: great potential as clinically translatable drug delivery vehicles? Nanomedicine (Lond). 2013; 8(10):1555-1557.

21. Münch G, Schicktanz D, Behme A, et al. Amino acid specificity of glycation and protein-AGE crosslinking reactivities determined with a dipeptide SPOT library. Nat Biotechnol. 1999;17(10):1006-1010.
22. Yang T, Wang Y, Ke HT, et al. Protein-nanoreactor-assisted synthesis of semiconductor nanocrystals for efficient cancer theranostics. $A d v$ Mater. 2016;28(28):5923-5930.

23. Moriyama Y, Ohta D, Hachiya K, Mitsui Y, Takeda K. Fluorescence behavior of tryptophan residues of bovine and human serum albumins in ionic surfactant solutions: a comparative study of the two and one tryptophan(s) of bovine and human albumins. J Protein Chem. 1996; 15(3):265-272.

24. Yuan HM, Xu C, Zhao Y, Yu BR, Cheng G, Xu FJ. Well-defined protein-based supramolecular nanoparticles with excellent MRI abilities for multifunctional delivery systems. Adv Funct Mater. 2016; 26(17):2855-2865.

25. Burt JL, Gutiérrez-Wing C, Miki-Yoshida M, José-Yacamán M. Noblemetal nanoparticles directly conjugated to globular proteins. Langmuir. 2004;20(26):11778-11783.

26. Huang P, Yang DP, Zhang CL, et al. Protein-directed one-pot synthesis of Ag microspheres with good biocompatibility and enhancement of radiation effects on gastric cancer cells. Nanoscale. 2011;3(9):3623-3626.

27. Liu HY, Zhang X, Wu XM, Jiang LP, Burda C, Zhu JJ. Rapid sonochemical synthesis of highly luminescent non-toxic AuNCs and Au@ $\mathrm{AgNCs}$ and $\mathrm{Cu}(\mathrm{II})$ sensing. Chem Commun (Camb). 2011;47(14): 4237-4239.

28. Wang Y, Wu YY, Liu YJ, et al. BSA-mediated synthesis of bismuth sulfide nanotheranostic agents for tumor multimodal imaging and thermoradiotherapy. Adv Funct Mater. 2016;26(29):5335-5344.

29. Wang Y, Yang T, Ke HT, et al. Smart albumin-biomineralized nanocomposites for multimodal imaging and photothermal tumor ablation. Adv Mater. 2015;27(26):3874-3882.

30. Novio F, Simmchen J, Vázquez-Mera N, et al. Coordination polymer nanoparticles in medicine. Coord Chem Rev. 2013;257(19-20): $2839-2847$.

31. Liu FY, He XX, Chen HD, Zhang JP, Zhang HM, Wang ZX. Gram-scale synthesis of coordination polymer nanodots with renal clearance properties for cancer theranostic applications. Nat Commun. 2015;6:8003.

32. Zeng JF, Cheng M, Wang Y, et al. pH-responsive Fe(III)-gallic acid nanoparticles for in vivo photoacoustic-imaging-guided photothermal therapy. Adv Healthc Mater. 2016;5(7):772-780.

33. Giftson JS, Jayanthi S, Nalini N. Chemopreventive efficacy of gallic acid, an antioxidant and anticarcinogenic polyphenol, against 1,2dimethyl hydrazine induced rat colon carcinogenesis. Invest New Drugs. 2010;28(3):251-259.

34. Li DJ, Zhu M, Xu C, Ji BM. Characterization of the baicalein-bovine serum albumin complex without or with $\mathrm{Cu}^{2+}$ or $\mathrm{Fe}^{3+}$ by spectroscopic approaches. Eur J Med Chem. 2011;46(2):588-599.

35. Li DJ, Zhu M, Xu C, Chen JJ, Ji BM. The effect of $\mathrm{Cu}^{2+}$ or $\mathrm{Fe}^{3+}$ on the noncovalent binding of rutin with bovine serum albumin by spectroscopic analysis. Spectrochim Acta A Mol Biomol Spectrosc. 2011; 78(1):74-79.

36. Das L, Guleria A, Adhikari S. Aqueous phase one-pot green synthesis of SnSe nanosheets in a protein matrix: negligible cytotoxicity and room temperature emission in the visible region. $R S C A d v .2015 ; 5(75)$ : 61390-61397.

37. Mohammed-Ziegler I, Billes F. Vibrational spectroscopic calculations on pyrogallol and gallic acid. Theochem. 2002;618(3):259-265.

38. Tan YX, Zhu LH, Niu HY, Cai YQ, Wu FC, Zhao XL. Synthesis of flower-shaped $\mathrm{ZrO}_{2}-\mathrm{C}$ composites for adsorptive removal of trichlorophenol from aqueous solution. RSC Adv. 2015;5(94):77175-77183.

39. Dai YL, Xiao HH, Liu JH, et al. In vivo multimodality imaging and cancer therapy by near-infrared light-triggered trans-platinum prodrug-conjugated upconverison [sic] nanoparticles. J Am Chem Soc. 2013;135(50):18920-18929. 


\section{Supplementary materials}

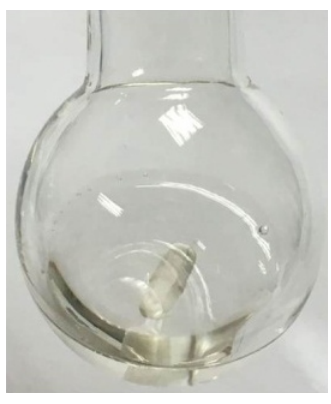

BSA

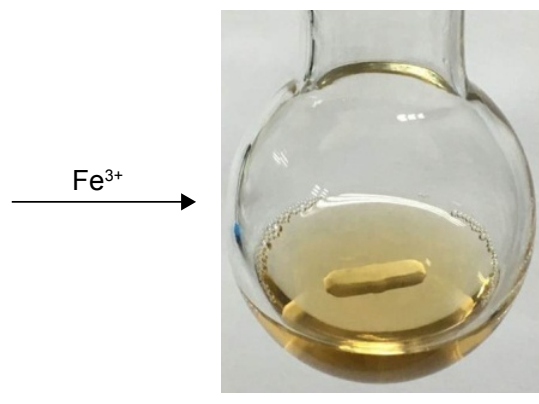

BSA-Fe ${ }^{3+}$ complex

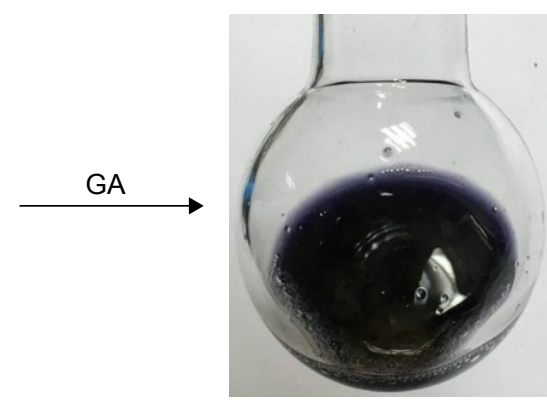

GA-Fe@BSA NPs

Figure SI Color changes at different stages in synthesis of GA-Fe@BSA nanoparticles.

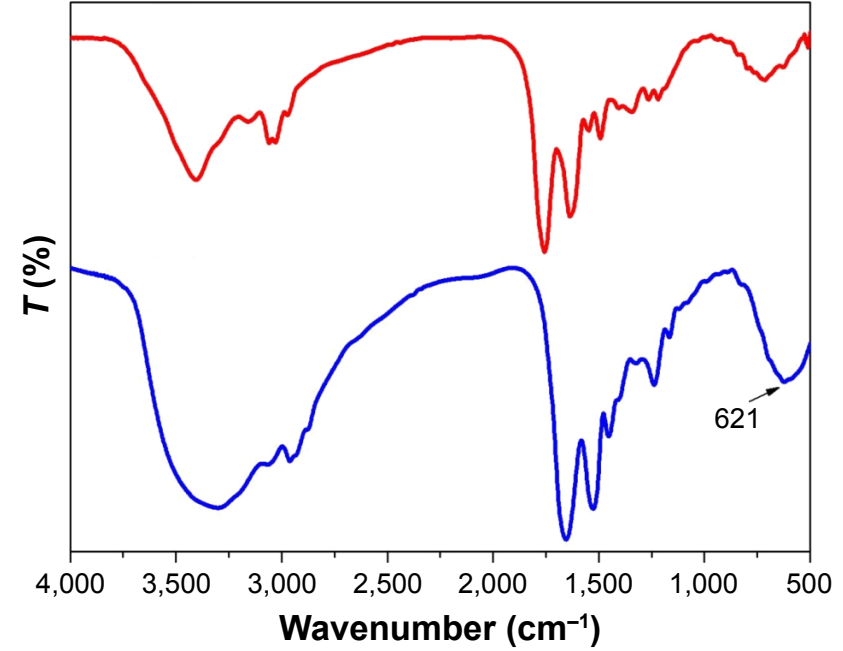

- BSA - BSA-Fe

Figure S2 FT-IR of the BSA (red color) and BSA-Fe complex (blue color).

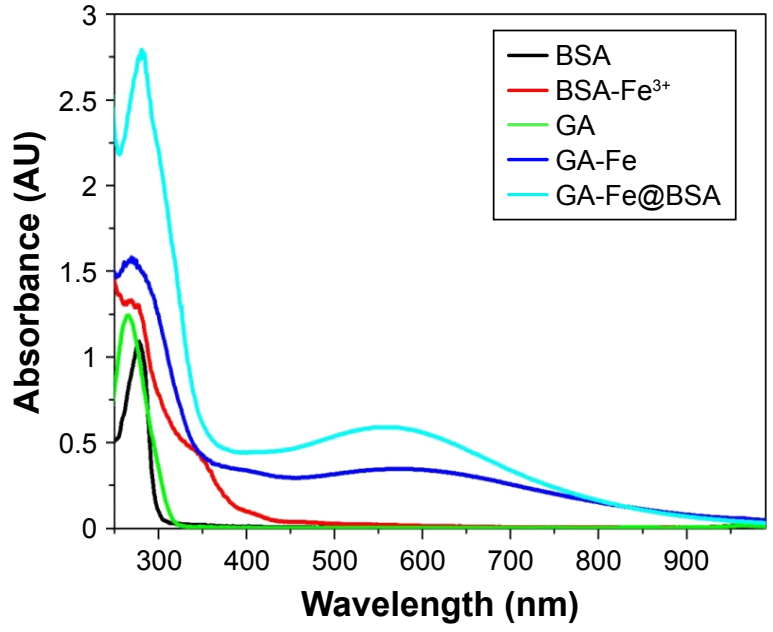

Figure S3 UV-vis-NIR absorption spectra of the BSA, BSA-Fe ${ }^{3+}$ complex, GA, GAFe and GA-Fe@BSA nanoparticles.

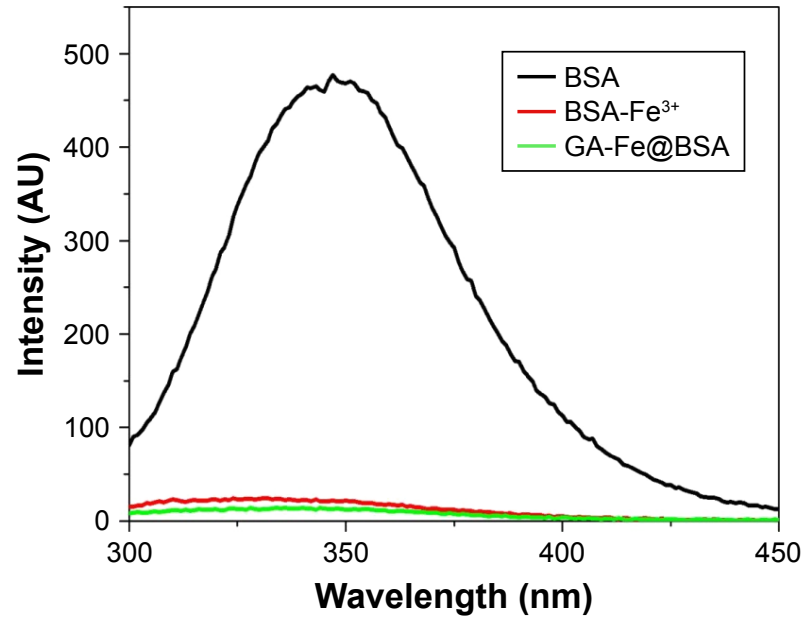

Figure S4 The fluorescence spectra of pure BSA, BSA after adding $\mathrm{Fe}^{3+}$ and GAFe@BSA nanoparticles.

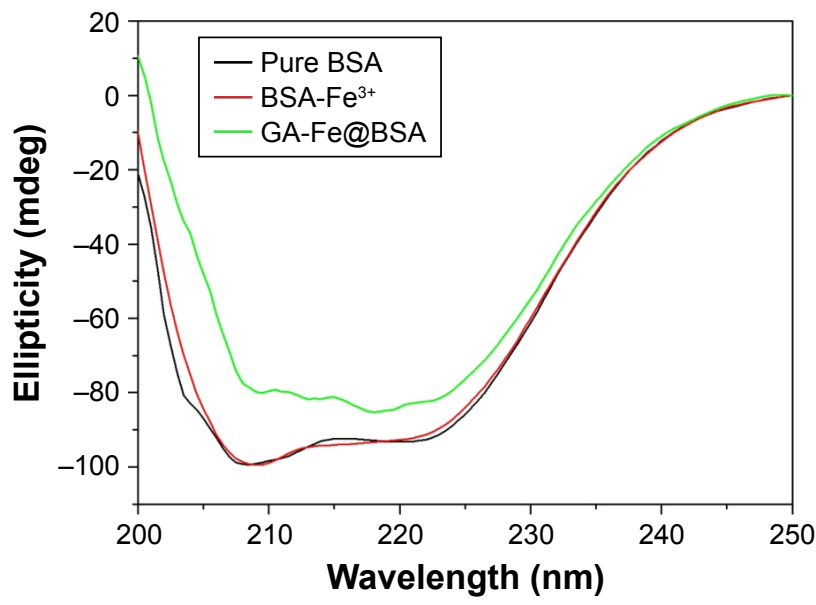

Figure S5 Circular dichroism spectra (recorded by BioLogic ALX250) of pure BSA, $\mathrm{BSA}^{-\mathrm{Fe}^{3+}}$ and GA-Fe@BSA nanoparticles. 


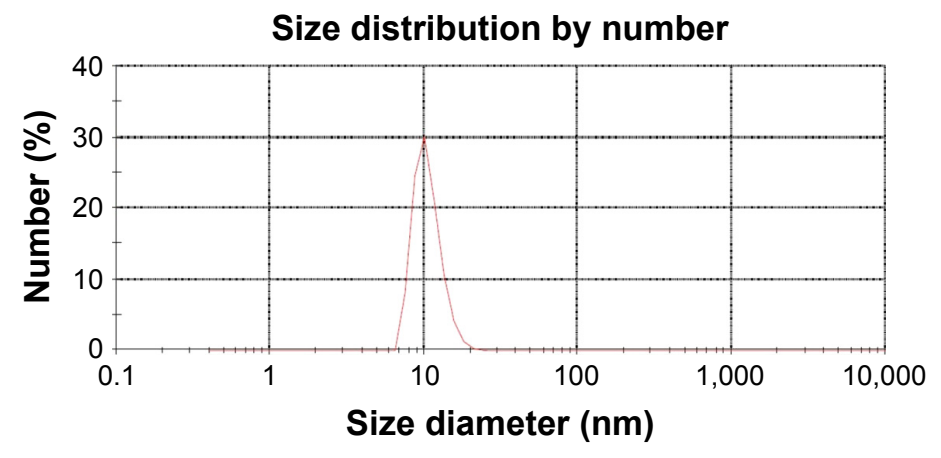

Record 291: GA-Fe@BSA 1

Figure S6 The primary data of dynamic light scattering analysis generated by the Malvern Zetasizer Nano ZS.
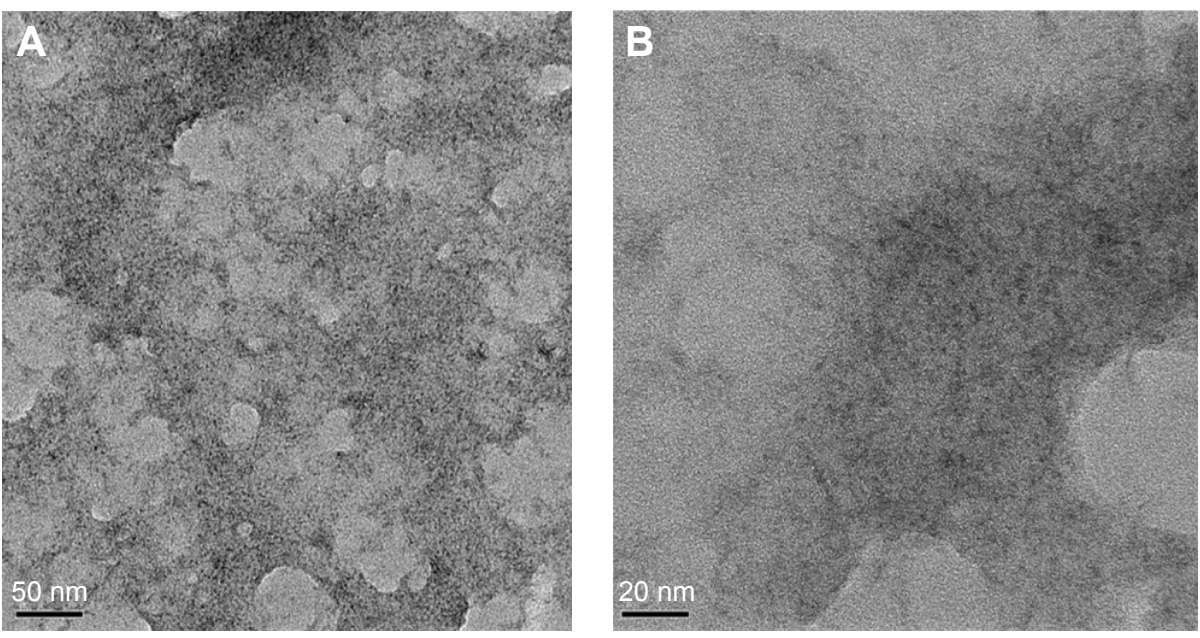

Figure S7 TEM images of the obtained with different magnification: (A) low magnification, (B) high magnification. Abbreviation: TEM, transmission electron microscopy.
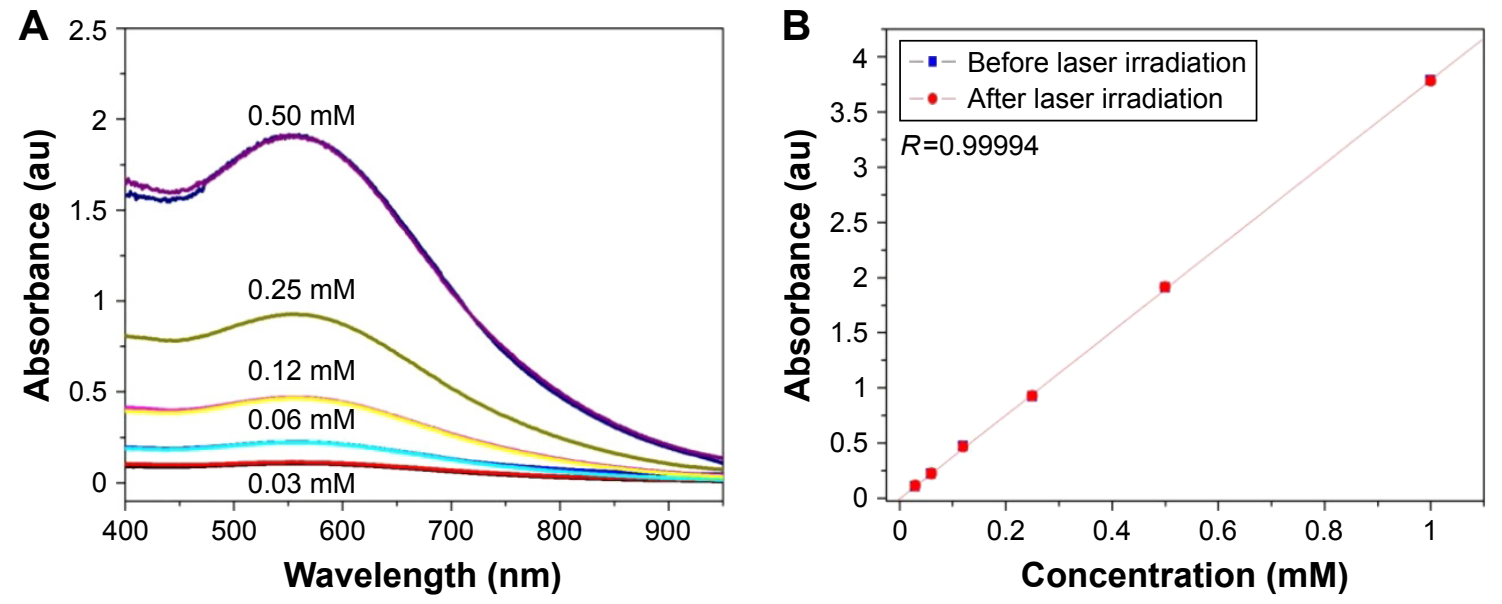

Figure S8 (A) UV-vis-NIR absorption spectra of the as prepared GA-Fe@BSA nanoparticles with different concentrations dispersed in water before and after the laser irradiation. (B) The absorption of the GA-Fe@BSA nanoparticles with different concentrations dispersed in water at $808 \mathrm{~nm}$ before and after the laser irradiation. 


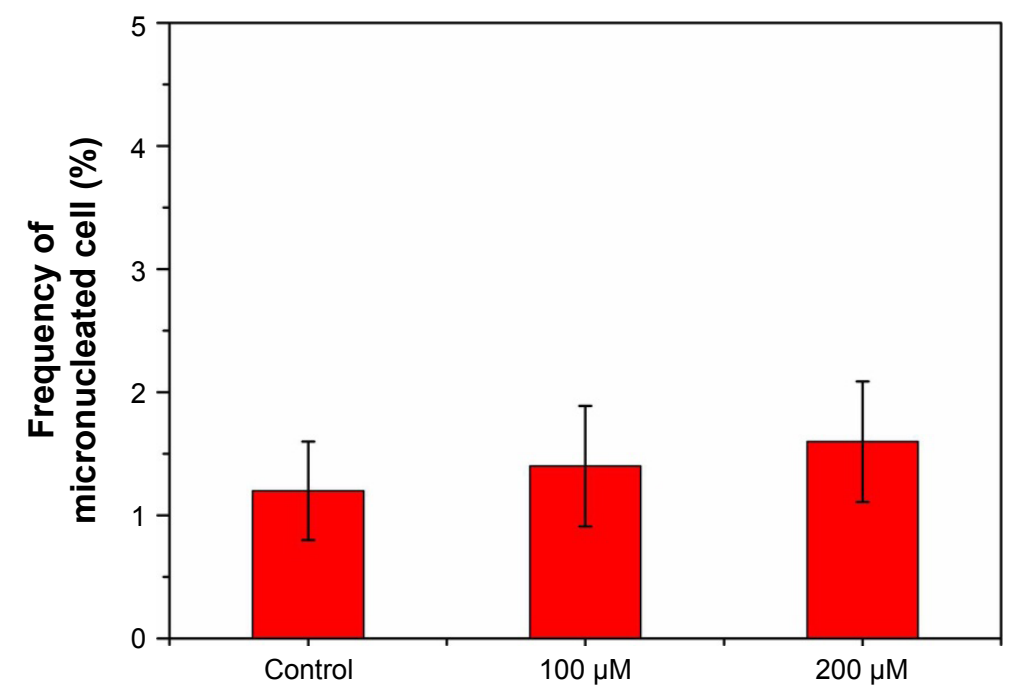

Figure S9 Frequency of micronucleated RAW cells incubated with GA-Fe@BSA. The values represent the mean of three experiments \pm SD. Each frequency is not significantly different $(p>0.05)$ from that of control cell in a Student's $t$-test (using the methods of AshaRani et al').
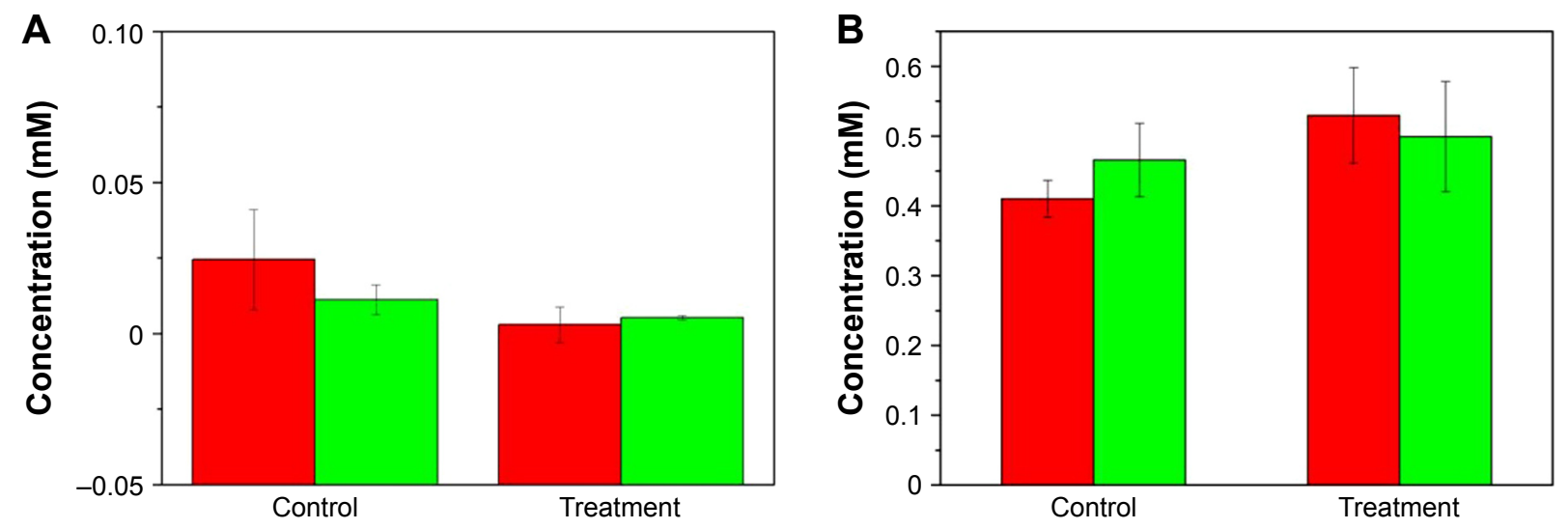

$\square 0 \mathrm{~h} \square 24 \mathrm{~h}$

Figure SI 0 The Iron concentration in the urine (A) and blood (B) of the mice administrated with PBS (control) and GA-Fe@BSA nanoparticles (treatment) at 0 and 24 h. 


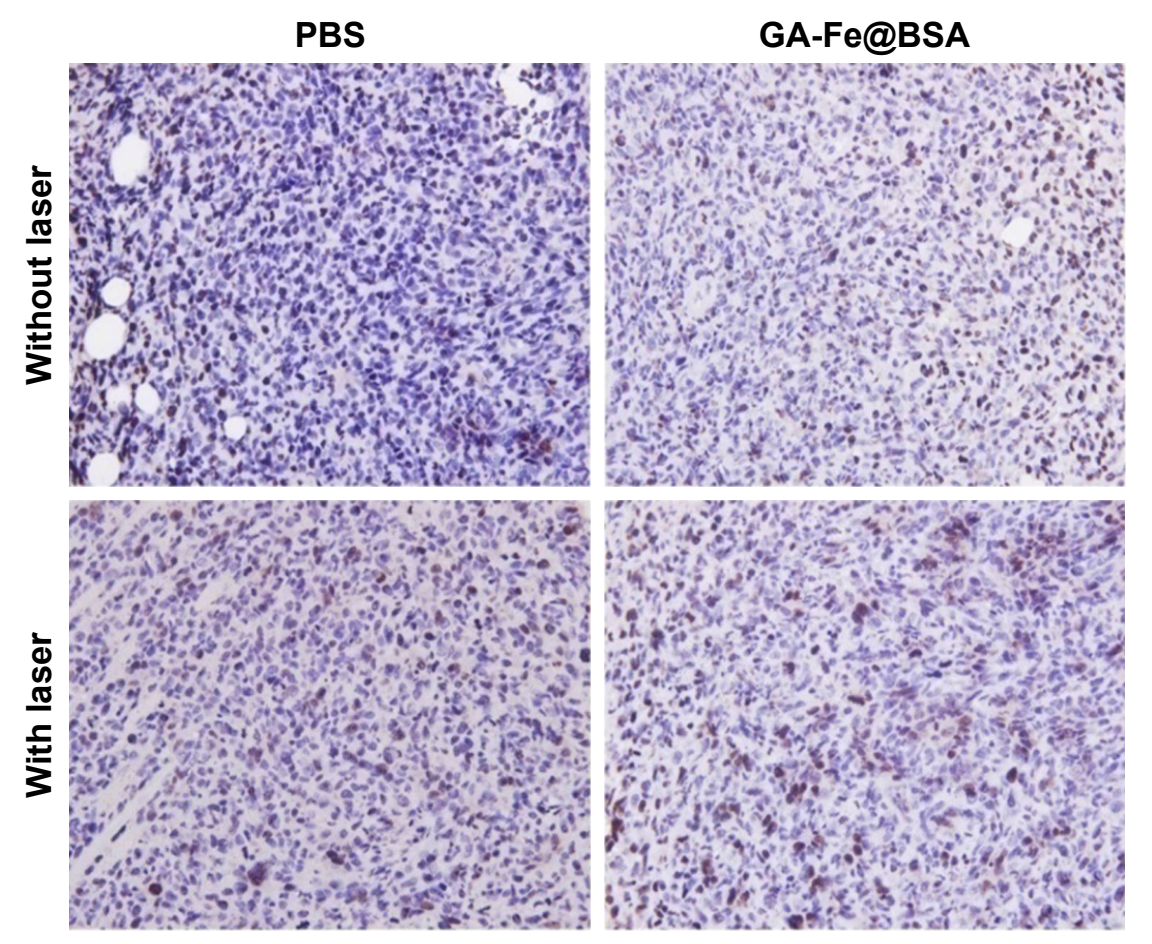

Figure SII Typical images tumor section with TUNEL staining for the group of PBS, PBS + laser, GA-Fe@BSA and GA-Fe@BSA + laser, respectively.

\section{Reference}

1. AshaRani PV, Low Kah Mun G, Hande MP, Valiyaveettil S. Cytotoxicity and genotoxicity of silver nanoparticles in human cells. ACS Nano. 2009;3(2):279-290.

\section{Publish your work in this journal}

The International Journal of Nanomedicine is an international, peerreviewed journal focusing on the application of nanotechnology in diagnostics, therapeutics, and drug delivery systems throughou the biomedical field. This journal is indexed on PubMed Central, MedLine, CAS, SciSearch ${ }^{\circledR}$, Current Contents ${ }^{\circledR} /$ Clinical Medicine,
Journal Citation Reports/Science Edition, EMBase, Scopus and the Elsevier Bibliographic databases. The manuscript management system is completely online and includes a very quick and fair peer-review system, which is all easy to use. Visit http://www.dovepress.com/ testimonials.php to read real quotes from published authors. 